\title{
Preparation of Mo, Co and iron group heterometallic clusters linked by cyclotetradeca-1,8-diyne ligand
}

\author{
Ming-Ann Hsu ${ }^{a}$, Wen-Yann Yeh ${ }^{\mathrm{a}, *}$, Gene-Hsiang Lee ${ }^{\mathrm{b}}$, Shie-Ming Peng ${ }^{\mathrm{b}, 1}$ \\ a Department of Chemistry, National Sun Yat-Sen University, Kaohsiung 804, Taiwan \\ ${ }^{\mathrm{b}}$ Department of Chemistry, National Taiwan University, Taipei 106, Taiwan
}

Received 11 May 1999; received in revised form 9 June 1999

\begin{abstract}
Thermal reactions of $\mathrm{Cp}_{2} \mathrm{Mo}_{2}(\mathrm{CO})_{4}\left(\mu-\eta^{2}-\mathrm{C}_{14} \mathrm{H}_{20}\right)$ (1) with $\mathrm{Fe}_{3}(\mathrm{CO})_{12}$ and $\mathrm{Ru}_{3}(\mathrm{CO})_{12}$ produce $\left[\mathrm{Cp}_{2} \mathrm{Mo}_{2}(\mathrm{CO})_{4}\right]\left(\mu, \mu_{3}-\eta^{2}, \eta^{2}\right.$ $\left.\mathrm{C}_{14} \mathrm{H}_{20}\right)\left[\mathrm{Fe}_{3}(\mathrm{CO})_{9}\right]$ (2) and $\left[\mathrm{Cp}_{2} \mathrm{Mo}_{2}(\mathrm{CO})_{4}\right]\left(\mu, \mu_{3}-\eta^{2}, \eta^{3}-\mathrm{C}_{14} \mathrm{H}_{19}\right)\left[(\mu-\mathrm{H}) \mathrm{Ru}_{3}(\mathrm{CO})_{9}\right]$ (3-allyl), respectively, while treatments of 1 with $\mathrm{Ru}_{3}(\mathrm{CO})_{10}(\mathrm{NCMe})_{2}$ and $\mathrm{Os}_{3}(\mathrm{CO})_{10}(\mathrm{NCMe})_{2}$ afford $\left[\mathrm{Cp}_{2} \mathrm{Mo}_{2}(\mathrm{CO})_{4}\right]\left(\mu, \mu_{3}-\eta^{2}, \eta^{3}-\mathrm{C}_{14} \mathrm{H}_{19}\right)\left[(\mu-\mathrm{H}) \mathrm{Ru}_{3}(\mathrm{CO})_{9}\right]$ (3-allenyl) and $\left[\mathrm{Cp}_{2} \mathrm{Mo}_{2}(\mathrm{CO})_{4}\right]\left(\mu, \mu_{3}-\eta^{2}, \eta^{2}-\mathrm{C}_{14} \mathrm{H}_{20}\right)\left[\mathrm{Os}_{3}(\mathrm{CO})_{10}\right](\mathbf{4})$, respectively. The allene moiety of 3 -allenyl rearranges upon heating to give the allyl complex 3-allyl. Compound 1 reacts with $\mathrm{Co}_{2}(\mathrm{CO})_{8}$ to produce $\left[\mathrm{Cp}_{2} \mathrm{Mo}_{2}(\mathrm{CO})_{4}\right]\left(\mu, \mu-\eta^{2}, \eta^{2}-\mathrm{C}_{14} \mathrm{H}_{20}\right)\left[\mathrm{Co}_{2}(\mathrm{CO})_{6}\right](5)$ and the reaction of $\mathrm{Os}_{3}(\mathrm{CO})_{10}\left(\mu_{3}-\eta^{2}-\mathrm{C}_{14} \mathrm{H}_{20}\right)$ with $\mathrm{Co}_{2}(\mathrm{CO})_{8}$ affords $\left[\mathrm{Os}_{3}(\mathrm{CO})_{10}\right]\left(\mu_{3}, \mu-\eta^{2}, \eta^{2}-\mathrm{C}_{14} \mathrm{H}_{20}\right)\left[\mathrm{Co}_{2}(\mathrm{CO})_{6}\right](\mathbf{6})$. The new compounds 2-6 have been characterized by mass, IR and NMR spectroscopy. The crystal structure of 4 reveals a bridging and a semibridging carbonyl connected to the $\mathrm{Os}_{3}$ cluster. (C) 1999 Elsevier Science S.A. All rights reserved.
\end{abstract}

Keywords: Molybdenum; Cobalt; Iron; Heterometallic clusters

\section{Introduction}

Heterometallic clusters offer organometallic chemists the opportunity to study the coordination and transformation of organic substrates with combinations of diverse metal atoms to lead to unique site selective reactions [1]. The interaction of alkynes with transition metal complexes has been under investigation for over 40 years and is still a subject of considerable research interest [2]. Recently, cycloalkadiynes [3] were shown to be versatile starting materials for synthesis of superphanes and cage compounds [4]. For instance, Gleiter and co-workers [5] carried out the reactions of cycloalkadiynes with $\mathrm{Fe}(\mathrm{CO})_{5}, \quad \mathrm{CpCo}(\mathrm{CO})_{2}$ and $\mathrm{Cp} * \mathrm{Co}\left(\mathrm{C}_{2} \mathrm{H}_{4}\right)_{2}$ to give metal-capped cyclobutadienophane and cyclobutadienosuperphane complexes. The coordination chemistry of cyclodiynes with transition metal clusters, however, has received relatively less attention. We previously described that cyclotetradeca1,8-diyne $\left(\mathrm{C}_{14} \mathrm{H}_{20}\right)$ reacted with $\mathrm{Fe}_{3}(\mathrm{CO})_{12}, \mathrm{Os}_{3}(\mathrm{CO})_{12}$

\footnotetext{
* Corresponding author. Fax: + 886-7-5253908.

${ }^{1}$ To whom inquiries concerning the X-ray crystallographic work should be addressed.
}

[6] and $\mathrm{Cp}_{2} \mathrm{Mo}_{2}(\mathrm{CO})_{6}$ [7] to afford complexes containing pendant alkyne moieties, which are capable of binding other metal fragments to form mixed-metal clusters or cluster oligomers. Presented in this paper are heterometallic carbonyl clusters of Mo, Co and iron group metals linked by the $\mathrm{C}_{14} \mathrm{H}_{20}$ ligand.

\section{Experimental}

\subsection{General methods}

All manipulations were carried out under an atmosphere of purified dinitrogen with standard Schlenk techniques [8]. $\mathrm{Co}_{2}(\mathrm{CO})_{8}, \mathrm{Ru}_{3}(\mathrm{CO})_{12}$ and $\mathrm{Fe}_{3}(\mathrm{CO})_{12}$ from Strem were used as received. $\mathrm{Os}_{3}(\mathrm{CO})_{12}$ was prepared from $\mathrm{OsO}_{4}$ and $\mathrm{CO}$ as described in the literature [9]. Cyclotetradeca-1,8-diyne $\left(\mathrm{C}_{14} \mathrm{H}_{20}\right)$ [10], $\mathrm{Cp}_{2} \mathrm{Mo}_{2}-$ (CO) $)_{4}\left(\mu-\eta^{2}-\mathrm{C}_{14} \mathrm{H}_{20}\right)(\mathbf{1})$ [7], $\mathrm{Os}_{3}(\mathrm{CO})_{10}\left(\mu_{3}-\eta^{2}-\mathrm{C}_{14} \mathrm{H}_{20}\right)$ [6], $\mathrm{Os}_{3}(\mathrm{CO})_{10}(\mathrm{NCMe})_{2}$ [11] and $\mathrm{Ru}_{3}(\mathrm{CO})_{10}(\mathrm{NCMe})_{2}$ [12] were prepared by literature methods. Solvents were dried over appropriate reagents under dinitrogen and distilled immediately before use [13]. Preparative thin-layer chromatographic (TLC) plates were 
prepared from silica gel (Merck). Infrared spectra were recorded with a $0.2 \mathrm{~mm}$ path-length $\mathrm{CaF}_{2}$ solution cell on a Hitachi I-2001 IR spectrometer. ${ }^{1} \mathrm{H}$ - and ${ }^{13} \mathrm{C}$ NMR spectra were obtained on a Varian VXR-300 spectrometer at 300 and $75.4 \mathrm{MHz}$, respectively. Fastatom-bombardment (FAB) mass spectra were recorded using a VG Blotch-5022 mass spectrometer. Elemental analyses were performed at the National Science Council Regional Instrumentation Center at National ChenKung University, Tainan, Taiwan.

\subsection{Reaction of 1 and $\mathrm{Fe}_{3}(\mathrm{CO})_{12}$}

$\mathrm{Cp}_{2} \mathrm{Mo}_{2}(\mathrm{CO})_{4}\left(\mu-\eta^{2}-\mathrm{C}_{14} \mathrm{H}_{20}\right)$ (1) (25 mg, $\left.0.04 \mathrm{mmol}\right)$ and $\mathrm{Fe}_{3}(\mathrm{CO})_{12}(61 \mathrm{mg}, 0.12 \mathrm{mmol})$ were dissolved in $n$-hexane $(20 \mathrm{ml})$ under an atmosphere of $\mathrm{N}_{2}$. The solution was heated to reflux for $1 \mathrm{~h}$, cooled to room temperature (r.t.), and the solvent removed on a rotary evaporator. The residue was then dissolved in $2 \mathrm{ml}$ of $\mathrm{CH}_{2} \mathrm{Cl}_{2}$ and subjected to TLC, with $3: 7 \mathrm{CH}_{2} \mathrm{Cl}_{2}-n$-hexane as eluant. The major brown band afforded $\left[\mathrm{Cp}_{2} \mathrm{Mo}_{2}(\mathrm{CO})_{4}\right]\left(\mu, \mu_{3}-\eta^{2}, \eta^{2}-\mathrm{C}_{14} \mathrm{H}_{20}\right)\left[\mathrm{Fe}_{3}(\mathrm{CO})_{9}\right]$ $\mathrm{mg}, 33 \%)$. MS (FAB) $m / z: 1046\left(\mathrm{M}^{+},{ }^{98} \mathrm{Mo}\right)$. IR $\left(\mathrm{CH}_{2} \mathrm{Cl}_{2}, v \mathrm{CO}\right): 2084 \mathrm{w}, 2024 \mathrm{vs}, 1996 \mathrm{~s}, 1984 \mathrm{sh}, 1942 \mathrm{~m}$, $1924 \mathrm{~m}, 1898 \mathrm{sh}, 1832 \mathrm{w} \mathrm{cm}{ }^{-1} .{ }^{1} \mathrm{H}-\mathrm{NMR}\left(\mathrm{CDCl}_{3}, 23^{\circ} \mathrm{C}\right)$ : 5.19 (s, Cp), $3.63(\mathrm{~m}), 2.77(\mathrm{~m}), 2.10(\mathrm{~m}), 1.79(\mathrm{~m}), 1.55$ (m), $1.24(\mathrm{~m}), 0.80\left(\mathrm{~m}, \mathrm{CH}_{2}\right)$ ppm. ${ }^{13} \mathrm{C}\left\{{ }^{1} \mathrm{H}\right\}-\mathrm{NMR}$ $\left(\mathrm{CDCl}_{3}, 23^{\circ} \mathrm{C}\right): 211.8(\mathrm{CO}), 207.7\left(\mathrm{C} \equiv \mathrm{C}-\mathrm{Fe}_{3}\right), 113.6$ $\left(\mathrm{C} \equiv \mathrm{C}-\mathrm{Mo}_{2}\right), 109.4\left(\mathrm{C} \equiv \mathrm{C}-\mathrm{Fe}_{3}\right), 90.4(\mathrm{Cp}), 46.0,30.1$, 28.7, 27.8, $26.6\left(\mathrm{CH}_{2}\right) \mathrm{ppm}$.

\subsection{Reaction of 1 and $\mathrm{Ru}_{3}(\mathrm{CO})_{12}$}

$\mathrm{Cp}_{2} \mathrm{Mo}_{2}(\mathrm{CO})_{4}\left(\mu-\eta^{2}-\mathrm{C}_{14} \mathrm{H}_{20}\right)$ (1) (28 mg, $\left.0.044 \mathrm{mmol}\right)$ and $\mathrm{Ru}_{3}(\mathrm{CO})_{12}(50 \mathrm{mg}, 0.078 \mathrm{mmol})$ were dissolved in $n$-heptane $(20 \mathrm{ml})$ under an atmosphere of $\mathrm{N}_{2}$. The solution was heated to reflux for $16 \mathrm{~h}$, cooled to r.t., and the solvent was removed under vacuum. The residue was dissolved in $\mathrm{CH}_{2} \mathrm{Cl}_{2}$ and subjected to TLC, eluting with 3:7 $\mathrm{CH}_{2} \mathrm{Cl}_{2}-n$-hexane. The major redbrown band afforded $\left[\mathrm{Cp}_{2} \mathrm{Mo}_{2}(\mathrm{CO})_{4}\right]\left(\mu, \mu_{3}-\eta^{2}, \eta^{3}-\right.$ $\left.\mathrm{C}_{14} \mathrm{H}_{19}\right)\left[(\mu-\mathrm{H}) \mathrm{Ru}_{3}(\mathrm{CO})_{9}\right]$ (3-allyl; $\left.27 \mathrm{mg}, 52 \%\right)$. MS (FAB) $m / z: 1184\left(\mathrm{M}^{+},{ }^{98} \mathrm{Mo},{ }^{102} \mathrm{Ru}\right)$. IR $\left(\mathrm{CH}_{2} \mathrm{Cl}_{2}\right.$, vCO): 2100m, 2072vs, 2044vs, 2020s, 2000s, 1986m, $1940 \mathrm{w}, 1920 \mathrm{~m}, 1910 \mathrm{~m}, 1898 \mathrm{~m}, 1828 \mathrm{w} \mathrm{cm}{ }^{-1} .{ }^{1} \mathrm{H}-\mathrm{NMR}$ $\left(\mathrm{CDCl}_{3}, 23^{\circ} \mathrm{C}\right): 7.80$ (s, allyl-H), 5.75 (s, Cp), 3.80-1.30 $\left(\mathrm{m}, \mathrm{CH}_{2}\right),-19.70(\mathrm{~s}, \mu-\mathrm{H}) \mathrm{ppm}$.

\subsection{Reaction of 1 and $\mathrm{Ru}_{3}(\mathrm{CO})_{10}(\mathrm{NCMe})_{2}$}

$\mathrm{Cp}_{2} \mathrm{Mo}_{2}(\mathrm{CO})_{4}\left(\mu-\eta^{2}-\mathrm{C}_{14} \mathrm{H}_{20}\right)$ (1) (25 mg, $\left.0.04 \mathrm{mmol}\right)$ and $\mathrm{Ru}_{3}(\mathrm{CO})_{10}(\mathrm{NCMe})_{2}(32 \mathrm{mg}, 0.048 \mathrm{mmol})$ were dissolved in $\mathrm{CH}_{2} \mathrm{Cl}_{2}(15 \mathrm{ml})$ under an atmosphere of $\mathrm{N}_{2}$. The solution was stirred at ambient temperature for $20 \mathrm{~min}$, and the solvent was removed on a rotary evaporator. The residue was applied on TLC, with 3:7
$\mathrm{CH}_{2} \mathrm{Cl}_{2}-n$-hexane as eluant. The major red-brown band gave $\left[\mathrm{Cp}_{2} \mathrm{Mo}_{2}(\mathrm{CO})_{4}\right]\left(\mu, \mu_{3}-\eta^{2}, \eta^{3}-\mathrm{C}_{14} \mathrm{H}_{19}\right)[(\mu-$ H) $\mathrm{Ru}_{3}(\mathrm{CO})_{9}$ ] (3-allenyl; $43 \mathrm{mg}, 88 \%$ ). MS (FAB) $\mathrm{m} / \mathrm{z}$ : $1184\left(\mathrm{M}^{+},{ }^{98} \mathrm{Mo},{ }^{102} \mathrm{Ru}\right)$. IR $\left(\mathrm{CH}_{2} \mathrm{Cl}_{2}, v \mathrm{CO}\right): 2100 \mathrm{~m}$, 2064vs, 2044vs, 2020s, 1996s, 1982s, 1944m, 1914s, 1904sh, $1834 \mathrm{~m} \mathrm{~cm}^{-1} .{ }^{1} \mathrm{H}-\mathrm{NMR}\left(\mathrm{CD}_{2} \mathrm{Cl}_{2}, 23^{\circ} \mathrm{C}\right): 5.20$ (s, Cp), $3.55(\mathrm{~d}, \mathrm{C}=\mathrm{C}=\mathrm{CH}), 3.10(\mathrm{~m}), 2.80(\mathrm{~m}), 2.15(\mathrm{~m})$, 1.90 (m), 1.70 (m), 1.45 (m), 1.30 (m), $1.15\left(\mathrm{~m}, \mathrm{CH}_{2}\right)$, $-20.57(\mathrm{~s}, \mu-\mathrm{H}) \mathrm{ppm}$.

\subsection{Reaction of 1 and $\mathrm{Os}_{3}(\mathrm{CO})_{10}(\mathrm{NCMe})_{2}$}

$\mathrm{Cp}_{2} \mathrm{Mo}_{2}(\mathrm{CO})_{4}\left(\mu-\eta^{2}-\mathrm{C}_{14} \mathrm{H}_{20}\right)$ (1) (100 mg, $\left.0.16 \mathrm{mmol}\right)$, $\mathrm{Os}_{3}(\mathrm{CO})_{10}(\mathrm{NCMe})_{2}(180 \mathrm{mg}, 0.19 \mathrm{mmol})$ and benzene $(30 \mathrm{ml})$ were added to a $50 \mathrm{ml}$ Schlenk flask under dinitrogen. The flask was placed in an oil bath at $65-70^{\circ} \mathrm{C}$ for $20 \mathrm{~min}$. Benzene was removed under vacuum and the residue was subjected to TLC with 1:1 $\mathrm{CH}_{2} \mathrm{Cl}_{2}-n$-hexane as eluant. The major brown band produced $\quad\left[\mathrm{Cp}_{2} \mathrm{Mo}_{2}(\mathrm{CO})_{4}\right]\left(\mu, \mu_{3}-\eta^{2}, \eta^{2}-\mathrm{C}_{14} \mathrm{H}_{20}\right)\left[\mathrm{Os}_{3}-\right.$ (CO) $\left.{ }_{10}\right](4 ; 215 \mathrm{mg}, 90 \%)$. MS (FAB) $\mathrm{m} / z: 1482\left(\mathrm{M}^{+}\right.$, $\left.{ }^{98} \mathrm{Mo},{ }^{192} \mathrm{Os}\right)$. IR $\left(\mathrm{CH}_{2} \mathrm{Cl}_{2}, v \mathrm{CO}\right): 2104 \mathrm{w}, 2060 \mathrm{vs}, 2044 \mathrm{~s}$, 2016s, 1994s, 1982sh, 1944m, 1912m, 1904sh, 1838w, $1828 \mathrm{sh} \mathrm{cm}^{-1} .{ }^{1} \mathrm{H}-\mathrm{NMR}\left(\mathrm{CDCl}_{3}, 23^{\circ} \mathrm{C}\right): 5.20$ (s, Cp), $2.92(\mathrm{~m}), 2.80(\mathrm{~m}), 2.17(\mathrm{~m}), 1.85(\mathrm{~m}), 1.70-1.30(\mathrm{~m}$, $\left.\mathrm{CH}_{2}\right)$ ppm. ${ }^{13} \mathrm{C}\left\{{ }^{1} \mathrm{H}\right\}-\mathrm{NMR}\left(\mathrm{CDCl}_{3},{ }^{2} 3^{\circ} \mathrm{C}\right): 177.0$ (br, $\mathrm{CO}), 148.4 \quad(\mathrm{C} \equiv \mathrm{C}-\mathrm{Os}), 111.5 \quad(\mathrm{C} \equiv \mathrm{C}-\mathrm{Mo}), 91.0 \quad(\mathrm{Cp})$, 49.2, 37.8, 33.0, 30.8, $28.3\left(\mathrm{CH}_{2}\right) \mathrm{ppm}$. Anal. Calc. for $\mathrm{C}_{38} \mathrm{H}_{30} \mathrm{Mo}_{2} \mathrm{O}_{14} \mathrm{Os}_{3}$ : C, 30.98; H, 2.05. Found: C, 30.83; $\mathrm{H}, 2.11 \%$.

\subsection{Reaction of 1 and $\mathrm{Co}_{2}(\mathrm{CO})_{8}$}

In a $50 \mathrm{ml}$ Schlenk flask, $\mathrm{Cp}_{2} \mathrm{Mo}_{2}(\mathrm{CO})_{4}\left(\mu-\eta^{2}-\mathrm{C}_{14} \mathrm{H}_{20}\right)$ (1) $(30 \mathrm{mg}, 0.048 \mathrm{mmol}), \mathrm{Co}_{2}(\mathrm{CO})_{8}(50 \mathrm{mg}, 0.146$ $\mathrm{mmol})$ and $\mathrm{CH}_{2} \mathrm{Cl}_{2}(20 \mathrm{ml})$ were introduced against a dinitrogen flow. The mixture was vigorously stirred at ambient temperature for $30 \mathrm{~min}$, and solvent was removed on a rotary evaporator. The residue was applied on TLC, eluting with 1:4 $\mathrm{CH}_{2} \mathrm{Cl}_{2}-n$-hexane. The major brown band was isolated, giving $\left[\mathrm{Cp}_{2} \mathrm{Mo}_{2}(\mathrm{CO})_{4}\right](\mu, \mu-$ $\left.\eta^{2}, \eta^{2}-\mathrm{C}_{14} \mathrm{H}_{20}\right)\left[\mathrm{Co}_{2}(\mathrm{CO})_{6}\right](5 ; 43 \mathrm{mg}, 95 \%)$. MS (FAB) $m / z: 912\left(\mathrm{M}^{+},{ }^{98} \mathrm{Mo}\right)$. IR $\left(\mathrm{CH}_{2} \mathrm{Cl}_{2}, v \mathrm{CO}\right): 2088 \mathrm{~m}$ 2044vs, 2014vs, 1982s, 1942s, 1920s, 1902sh, $1834 \mathrm{~m}$ $\mathrm{cm}^{-1} .{ }^{1} \mathrm{H}-\mathrm{NMR}\left(\mathrm{CDCl}_{3}, 28^{\circ} \mathrm{C}\right): 5.21$ (s, Cp), 2.93 (br, $\mathrm{CH}_{2}$ ), 1.69 (br, $\mathrm{CH}_{2}$ ), 1.50 (br, $\mathrm{CH}_{2}$ ) ppm.

\subsection{Preparation of \\ $\left[\mathrm{Os}_{3}(\mathrm{CO})_{10}\right]\left(\mu_{3}, \mu-\eta^{2}, \eta^{2}-\mathrm{C}_{14} \mathrm{H}_{20}\right)\left[\mathrm{Co}_{2}(\mathrm{CO})_{6}\right]$ (6)}

$\mathrm{Os}_{3}(\mathrm{CO})_{10}\left(\mu_{3}-\eta^{2}-\mathrm{C}_{14} \mathrm{H}_{20}\right)(40 \mathrm{mg}, 0.039 \mathrm{mmol})$ was dissolved in $n$-hexane $(20 \mathrm{ml})$ under an atmosphere of dinitrogen, and $\mathrm{Co}_{2}(\mathrm{CO})_{8}(20 \mathrm{mg}, 0.058 \mathrm{mmol})$ was added against a dinitrogen flow. The mixture was stirred at ambient temperature for $30 \mathrm{~min}$, and solvent was removed on a rotary evaporator. The residue was 
subjected to TLC, eluting with $n$-hexane. The major brown band afforded $\left[\mathrm{Os}_{3}(\mathrm{CO})_{10}\right]\left(\mu_{3}, \mu-\eta^{2}, \eta^{2}-\mathrm{C}_{14^{-}}\right.$ $\left.\mathrm{H}_{20}\right)\left[\mathrm{Co}_{2}(\mathrm{CO})_{6}\right](6 ; 45 \mathrm{mg}, 88 \%)$. MS (FAB) $m / z$ : $1330\left(\mathrm{M}^{+},{ }^{192} \mathrm{Os}\right)$. IR $\left(\mathrm{CH}_{2} \mathrm{Cl}_{2}, v \mathrm{CO}\right): 2102 \mathrm{w}, 2090 \mathrm{~m}$, 2062s, 2046vs, 2018s, 2000sh, 1985sh, 1845vw $\mathrm{cm}^{-1}$. ${ }^{1} \mathrm{H}-\mathrm{NMR}\left(\mathrm{CDCl}_{3}, 23^{\circ} \mathrm{C}\right): 2.72$ (br, $\left.\mathrm{CH}_{2}\right), 2.51$ (br, $\mathrm{CH}_{2}$ ), 2.04 (br, $\mathrm{CH}_{2}$ ), 1.62 (br, $\mathrm{CH}_{2}$ ), 1.39 (br, $\mathrm{CH}_{2}$ ), 1.16 (br, $\left.\mathrm{CH}_{2}\right)$ ppm. ${ }^{13} \mathrm{C}\left\{{ }^{1} \mathrm{H}\right\}-\mathrm{NMR}\left(\mathrm{CDCl}_{3}, 23^{\circ} \mathrm{C}\right)$ : 200.7 (br, $\mathrm{Co}-\mathrm{CO}$ ), 177.0 (br, Os-CO), 148.3 $\left(\mathrm{C} \equiv \mathrm{C}-\mathrm{Os}_{3}\right), 98.2\left(\mathrm{C} \equiv \mathrm{C}-\mathrm{Co}_{2}\right), 49.2,34.7,33.0,30.9,28.0$ $\left(\mathrm{CH}_{2}\right) \mathrm{ppm}$.

\subsection{Structure determination for 3-allyl and $\mathbf{4}$}

A crystal of $\left[\mathrm{Cp}_{2} \mathrm{Mo}_{2}(\mathrm{CO})_{4}\right]\left(\mu, \mu_{3}-\eta^{2}, \eta^{3}-\mathrm{C}_{14} \mathrm{H}_{19}\right)$ $\left[(\mu-\mathrm{H}) \mathrm{Ru}_{3}(\mathrm{CO})_{9}\right] \quad(3$-allyl; ca. $0.10 \times 0.10 \times 0.01 \mathrm{~mm})$ and a crystal of $\left[\mathrm{Cp}_{2} \mathrm{Mo}_{2}(\mathrm{CO})_{4}\right]\left(\mu, \mu_{3}-\eta^{2}, \eta^{2}-\mathrm{C}_{14} \mathrm{H}_{20}\right)\left[\mathrm{Os}_{3}-\right.$ $\left.(\mathrm{CO})_{10}\right] \quad(4$; ca. $0.40 \times 0.20 \times 0.04 \mathrm{~mm})$ were each mounted in a thin-walled glass capillary and aligned on the Siemens SMART-CCD diffractometer with graphite-monochromated $\mathrm{Mo}-\mathrm{K}_{\alpha}$ radiation $(\lambda=$ $0.71073 \AA$ ). The $\theta$ range for data collection was 0.77 $26.37^{\circ}$ for 3-allyl and $0.68-26.37^{\circ}$ for 4 . A total of 18686 reflections were measured, and 8009 reflections $\left(R_{\text {int }}=0.0850\right)$ were unique for 3-allyl, while 25893 reflections were measured and 8667 reflections $\left(R_{\text {int }}=\right.$ 0.0613 ) were unique for 4. SADABS [14] absorption corrections were made for 3-allyl $\left(T_{\min }=0.8622\right.$, $\left.T_{\max }=0.7299\right)$ and $4\left(T_{\min }=0.6948, T_{\max }=0.3520\right)$. The structures were solved by the direct method and

Table 1

Crystallographic data for $\left[\mathrm{Cp}_{2} \mathrm{Mo}_{2}(\mathrm{CO})_{4}\right]\left(\mu, \mu_{3}-\eta^{2}, \eta^{3}-\mathrm{C}_{14} \mathrm{H}_{19}\right)[(\mu-$ $\left.\mathrm{H}) \mathrm{Ru}_{3}(\mathrm{CO})_{9}\right]$ (3-allyl) and $\left[\mathrm{Cp}_{2} \mathrm{Mo}_{2}(\mathrm{CO})_{4}\right]\left(\mu, \mu_{3}-\eta^{2}, \eta^{2}\right.$ $\left.\mathrm{C}_{14} \mathrm{H}_{20}\right)\left[\mathrm{Os}_{3}(\mathrm{CO})_{10}\right](4)$

\begin{tabular}{|c|c|c|}
\hline & 3-allyl & 4 \\
\hline Formula & $\mathrm{C}_{37} \mathrm{H}_{30} \mathrm{Mo}_{2} \mathrm{O}_{13} \mathrm{Ru}_{3}$ & $\mathrm{C}_{38} \mathrm{H}_{30} \mathrm{Mo}_{2} \mathrm{O}_{14} \mathrm{Os}$ \\
\hline$F_{\mathrm{w}}$ & 1177.69 & $\begin{array}{l}3 \\
1515.56\end{array}$ \\
\hline Crystal solvent & & $0.5 \mathrm{CH}_{2} \mathrm{Cl}_{2}$ \\
\hline$T(\mathrm{~K})$ & $295(2)$ & $295(2)$ \\
\hline Crystal system & Monoclinic & Triclinic \\
\hline Space group & $C 2 / c$ & $P \overline{1}$ \\
\hline \multicolumn{3}{|l|}{ Unit cell dimensions } \\
\hline$a(\AA)$ & $52.7221(1)$ & $8.4722(1)$ \\
\hline$b(\AA)$ & $9.7332(1)$ & $9.2362(1)$ \\
\hline$c(\AA)$ & $15.5231(3)$ & $30.1618(2)$ \\
\hline$\alpha\left({ }^{\circ}\right)$ & 90 & $93.377(1)$ \\
\hline$\beta\left(^{\circ}\right)$ & $90.377(1)$ & $95.311(1)$ \\
\hline$\gamma\left({ }^{\circ}\right)$ & 90 & $114.531(1)$ \\
\hline$V\left(\AA^{3}\right)$ & $7965.6(2)$ & $2125.55(4)$ \\
\hline$Z$ & 8 & 2 \\
\hline$D_{\text {calc }}\left(\mathrm{g} \mathrm{cm}^{-3}\right)$ & 1.962 & 2.368 \\
\hline$\mu\left(\mathrm{mm}^{-1}\right)$ & 1.786 & 9.635 \\
\hline$R_{1} / w R_{2}^{a}$ & $0.0600 / 0.1042$ & $0.0366 / 0.0860$ \\
\hline Goodness-of-fit on $F^{2}$ & 1.054 & 1.059 \\
\hline
\end{tabular}

\footnotetext{
$\left.{ }^{\mathrm{a}} R_{1}=\Sigma|| F_{\mathrm{o}}|-| F_{\mathrm{c}}|| / \Sigma\left|F_{\mathrm{o}}\right| ; w R_{2}=\left\{\Sigma\left[w\left(\left|F_{\mathrm{o}}\right|^{2}-\left|F_{\mathrm{c}}\right|^{2}\right)^{2}\right] / \Sigma w\left|F_{\mathrm{o}}\right|^{4}\right]\right\}^{1 / 2}$
}

Table 2

Atomic coordinates $\left(\times 10^{4}\right)$ and equivalent isotropic displacement parameters $\left(\AA^{2} \times 10^{3}\right)$ for 3-allyl

\begin{tabular}{|c|c|c|c|c|}
\hline Atom & $x$ & $y$ & $z$ & $U_{\text {eq }}$ \\
\hline $\mathrm{Ru}(1)$ & $1748(1)$ & $1568(1)$ & $2147(1)$ & $40(1)$ \\
\hline $\mathrm{Ru}(2)$ & $2018(1)$ & $1585(1)$ & $3697(1)$ & $34(1)$ \\
\hline $\mathrm{Ru}(3)$ & $1923(1)$ & 4144(1) & $2959(1)$ & $38(1)$ \\
\hline $\operatorname{Mo}(1)$ & $377(1)$ & 1984(1) & $4760(1)$ & $33(1)$ \\
\hline $\operatorname{Mo}(2)$ & $678(1)$ & $2475(1)$ & $6362(1)$ & $34(1)$ \\
\hline $\mathrm{C}(1)$ & $1434(3)$ & 1733(13) & $1518(8)$ & $63(3)$ \\
\hline $\mathrm{C}(2)$ & $1797(2)$ & $-322(13)$ & $1895(7)$ & $53(3)$ \\
\hline $\mathrm{C}(3)$ & $1971(2)$ & 1952(13) & $1179(7)$ & $68(4)$ \\
\hline $\mathrm{C}(4)$ & $2060(2)$ & $-374(13)$ & $3706(8)$ & $59(3)$ \\
\hline$C(5)$ & $2300(2)$ & 1989(11) & $2982(7)$ & $51(3)$ \\
\hline$C(6)$ & $2180(2)$ & 1971(12) & 4796(8) & $53(3)$ \\
\hline$C(7)$ & $2133(2)$ & $4556(11)$ & 1942(8) & $53(3)$ \\
\hline $\mathrm{C}(8)$ & $2157(2)$ & $5113(11)$ & $3678(7)$ & $47(3)$ \\
\hline $\mathrm{C}(9)$ & 1699(2) & $5609(13)$ & $2765(9)$ & $68(4)$ \\
\hline $\mathrm{C}(10)$ & $447(2)$ & $37(12)$ & $4663(7)$ & $42(3)$ \\
\hline $\mathrm{C}(11)$ & 191(2) & $1597(11)$ & $5816(6)$ & $43(3)$ \\
\hline$C(12)$ & $644(2)$ & 490(13) & $6581(6)$ & $44(3)$ \\
\hline $\mathrm{C}(13)$ & $1042(2)$ & $2085(11)$ & $6444(6)$ & $40(2)$ \\
\hline$C(14)$ & $1605(2)$ & 1131(9) & $3369(6)$ & $36(2)$ \\
\hline$C(15)$ & $1616(2)$ & 2094(9) & $4050(6)$ & $33(2)$ \\
\hline$C(16)$ & $1752(2)$ & $3334(9)$ & $4040(6)$ & $33(2)$ \\
\hline $\mathrm{C}(17)$ & $1739(2)$ & 4141(10) & $4886(7)$ & $46(3)$ \\
\hline $\mathrm{C}(18)$ & $1562(2)$ & $5405(13)$ & $4839(8)$ & $73(4)$ \\
\hline $\mathrm{C}(19)$ & $1298(2)$ & $5239(16)$ & 4471(9) & $84(4)$ \\
\hline $\mathrm{C}(20)$ & $1137(3)$ & $4443(15)$ & 4983(8) & $91(5)$ \\
\hline $\mathrm{C}(21)$ & $849(2)$ & $4319(12)$ & $4650(7)$ & $56(3)$ \\
\hline $\mathrm{C}(22)$ & $729(2)$ & $3071(11)$ & $5029(6)$ & $37(2)$ \\
\hline$C(23)$ & $783(2)$ & $1722(10)$ & $5024(6)$ & $33(2)$ \\
\hline$C(24)$ & $984(2)$ & 804(11) & $4671(6)$ & $42(3)$ \\
\hline$C(25)$ & $1019(2)$ & 973(11) & $3700(6)$ & $43(3)$ \\
\hline$C(26)$ & $1181(2)$ & $-125(12)$ & $3293(7)$ & $54(3)$ \\
\hline$C(27)$ & $1459(2)$ & $-172(10)$ & $3569(7)$ & $50(3)$ \\
\hline $\mathrm{C}(28)$ & $4(2)$ & 2106(15) & $3968(7)$ & $60(4)$ \\
\hline C(29) & $203(2)$ & 1891(14) & $3376(7)$ & $61(3)$ \\
\hline$C(30)$ & $362(2)$ & $3029(16)$ & $3391(8)$ & $70(4)$ \\
\hline $\mathrm{C}(31)$ & $255(3)$ & $3984(14)$ & $3977(9)$ & $73(4)$ \\
\hline $\mathrm{C}(32)$ & $37(2)$ & $3396(14)$ & $4316(8)$ & $65(4)$ \\
\hline $\mathrm{C}(33)$ & $369(2)$ & 4049(13) & $6891(7)$ & $54(3)$ \\
\hline$C(34)$ & $431(2)$ & $3127(12)$ & $7526(7)$ & $54(3)$ \\
\hline$C(35)$ & $685(2)$ & $3303(13)$ & $7746(7)$ & $57(3)$ \\
\hline$C(36)$ & $784(2)$ & $4352(13)$ & $7215(8)$ & $62(4)$ \\
\hline$C(37)$ & $586(3)$ & $4788(13)$ & $6682(7)$ & 64(3) \\
\hline $\mathrm{O}(1)$ & $1246(2)$ & 1813(12) & $1173(7)$ & $120(4)$ \\
\hline $\mathrm{O}(2)$ & $1834(2)$ & $-1436(10)$ & $1729(6)$ & 84(3) \\
\hline $\mathrm{O}(3)$ & $2101(2)$ & 2084(11) & 604(6) & $109(4)$ \\
\hline $\mathrm{O}(4)$ & $2090(2)$ & $-1519(9)$ & $3686(7)$ & 99(3) \\
\hline $\mathrm{O}(5)$ & $2480(2)$ & $2102(10)$ & $2591(6)$ & $81(3)$ \\
\hline $\mathrm{O}(6)$ & $2276(2)$ & 1879(11) & $5446(6)$ & $85(3)$ \\
\hline $\mathrm{O}(7)$ & $2255(2)$ & $4817(10)$ & $1372(6)$ & $88(3)$ \\
\hline $\mathrm{O}(8)$ & $2301(2)$ & $5644(9)$ & $4117(5)$ & $68(2)$ \\
\hline $\mathrm{O}(9)$ & $1547(2)$ & $6452(10)$ & $2679(8)$ & $106(4)$ \\
\hline $\mathrm{O}(10)$ & 496(1) & $-1127(8)$ & $4570(6)$ & $66(2)$ \\
\hline $\mathrm{O}(11)$ & 47(1) & 1352(9) & $6365(5)$ & $64(2)$ \\
\hline $\mathrm{O}(12)$ & $625(2)$ & $-671(9)$ & $6747(6)$ & $72(3)$ \\
\hline $\mathrm{O}(13)$ & $1256(1)$ & 1827(8) & $6493(4)$ & $53(2)$ \\
\hline
\end{tabular}

refined by full-matrix least-squares on $F^{2}$. The program used was the SHELXTL package. The data collection and refinement parameters are collected in Table 1. Atomic 
positional parameters of $\mathbf{3}$-allyl and $\mathbf{4}$ are given in Tables 2 and 3 , respectively.

Table 3

Atomic coordinates $\left(\times 10^{4}\right)$ and equivalent isotropic displacement parameters $\left(\AA^{2} \times 10^{3}\right)$ for 4

\begin{tabular}{|c|c|c|c|c|}
\hline Atom & $x$ & $y$ & $z$ & $U_{\text {eq }}$ \\
\hline Os(1) & $8783(1)$ & $5454(1)$ & $3897(1)$ & $31(1)$ \\
\hline Os(2) & $6574(1)$ & $6847(1)$ & $4095(1)$ & $35(1)$ \\
\hline Os(3) & $9911(1)$ & $8817(1)$ & $3933(1)$ & $39(1)$ \\
\hline $\operatorname{Mo}(1)$ & 282(1) & $4872(1)$ & $1448(1)$ & $46(1)$ \\
\hline $\operatorname{Mo}(2)$ & $3128(1)$ & $7087(1)$ & 992(1) & $42(1)$ \\
\hline$C(1)$ & $9100(10)$ & $3883(9)$ & 3513(3) & $45(2)$ \\
\hline$C(2)$ & $6919(10)$ & $3868(9)$ & $4163(3)$ & $47(2)$ \\
\hline$C(3)$ & $10448(11)$ & $5567(10)$ & $4408(3)$ & $51(2)$ \\
\hline $\mathrm{C}(4)$ & 4283(10) & $5123(9)$ & $4003(2)$ & $45(2)$ \\
\hline$C(5)$ & $6927(10)$ & $6916(9)$ & $4740(3)$ & $46(2)$ \\
\hline $\mathrm{C}(6)$ & $5594(10)$ & $8356(10)$ & $4126(3)$ & $49(2)$ \\
\hline$C(7)$ & $11561(10)$ & 9300(9) & $4478(3)$ & $52(2)$ \\
\hline $\mathrm{C}(8)$ & 9326(10) & $10492(9)$ & $4146(3)$ & $48(2)$ \\
\hline $\mathrm{C}(9)$ & $11538(11)$ & $10147(10)$ & $3570(3)$ & $59(2)$ \\
\hline $\mathrm{C}(10)$ & $10849(10)$ & 7068(9) & $3604(3)$ & $43(2)$ \\
\hline $\mathrm{C}(11)$ & $321(11)$ & $4878(14)$ & $2106(3)$ & $73(3)$ \\
\hline $\mathrm{C}(12)$ & $-427(12)$ & $6622(14)$ & $1558(3)$ & $71(3)$ \\
\hline $\mathrm{C}(13)$ & 798(11) & $6510(10)$ & $682(3)$ & $56(2)$ \\
\hline $\mathrm{C}(14)$ & $3064(11)$ & $9062(11)$ & $1236(3)$ & $59(2)$ \\
\hline$C(15)$ & $7098(8)$ & $5986(8)$ & $3430(2)$ & $30(1)$ \\
\hline$C(16)$ & $7689(9)$ & $7662(8)$ & $3444(2)$ & $39(2)$ \\
\hline$C(17)$ & $6781(15)$ & $8526(14)$ & $3140(4)$ & $104(5)$ \\
\hline $\mathrm{C}(18)$ & $7811(16)$ & $9264(15)$ & $2805(4)$ & $96(4)$ \\
\hline C(19) & $6733(18)$ & $10121(11)$ & $2543(4)$ & $120(6)$ \\
\hline $\mathrm{C}(20)$ & $5660(14)$ & 9049(11) & $2126(4)$ & 93(4) \\
\hline$C(21)$ & $3746(12)$ & $8024(10)$ & $2128(3)$ & $61(2)$ \\
\hline $\mathrm{C}(22)$ & 2953(9) & $6902(9)$ & $1712(2)$ & $41(2)$ \\
\hline $\mathrm{C}(23)$ & $3079(8)$ & $5587(8)$ & $1531(2)$ & $38(2)$ \\
\hline $\mathrm{C}(24)$ & 4113(9) & $4697(9)$ & $1689(2)$ & $43(2)$ \\
\hline $\mathrm{C}(25)$ & $3524(10)$ & $3834(10)$ & 2099(3) & $51(2)$ \\
\hline$C(26)$ & $4780(12)$ & $3212(9)$ & $2320(3)$ & $55(2)$ \\
\hline $\mathrm{C}(27)$ & $6259(9)$ & $4452(10)$ & $2655(2)$ & $45(2)$ \\
\hline $\mathrm{C}(28)$ & $5628(9)$ & $4799(9)$ & $3092(2)$ & $42(2)$ \\
\hline C(29) & $-47(13)$ & $2347(11)$ & $1122(4)$ & $71(3)$ \\
\hline $\mathrm{C}(30)$ & $-701(11)$ & $3027(10)$ & 793(3) & $62(2)$ \\
\hline $\mathrm{C}(31)$ & $-2121(11)$ & $3242(12)$ & $930(3)$ & $69(3)$ \\
\hline $\mathrm{C}(32)$ & $-2372(12)$ & $2659(14)$ & $1356(4)$ & $90(4)$ \\
\hline C(33) & $-1051(14)$ & $2120(12)$ & $1467(4)$ & $84(4)$ \\
\hline$C(34)$ & $5015(22)$ & $6090(20)$ & $700(5)$ & $105(5)$ \\
\hline$C(35)$ & $3882(14)$ & $6167(16)$ & $338(4)$ & 91(4) \\
\hline$C(36)$ & $4224(16)$ & $7754(17)$ & $319(3)$ & $88(3)$ \\
\hline $\mathrm{C}(37)$ & $5529(19)$ & $8594(18)$ & $644(5)$ & $123(6)$ \\
\hline $\mathrm{C}(38)$ & $5994(17)$ & $7661(31)$ & $887(4)$ & $135(8)$ \\
\hline $\mathrm{O}(1)$ & $9318(9)$ & 2971(7) & $3296(2)$ & $72(2)$ \\
\hline $\mathrm{O}(2)$ & 5911(9) & $2817(7)$ & $4303(3)$ & $76(2)$ \\
\hline $\mathrm{O}(3)$ & $11493(9)$ & $5722(9)$ & $4696(2)$ & $78(2)$ \\
\hline $\mathrm{O}(4)$ & 2929(7) & $4112(7)$ & $3939(2)$ & $62(2)$ \\
\hline $\mathrm{O}(5)$ & $7191(10)$ & $6934(9)$ & $5109(2)$ & $80(2)$ \\
\hline $\mathrm{O}(6)$ & $5000(8)$ & $9254(7)$ & $4144(3)$ & $73(2)$ \\
\hline $\mathrm{O}(7)$ & $12434(9)$ & 9513(9) & $4808(2)$ & $81(2)$ \\
\hline $\mathrm{O}(8)$ & $9051(8)$ & $11540(7)$ & $4267(2)$ & $69(2)$ \\
\hline $\mathrm{O}(9)$ & $12477(11)$ & $10882(9)$ & $3346(3)$ & $103(2)$ \\
\hline $\mathrm{O}(10)$ & $11953(8)$ & $7211(8)$ & $3401(2)$ & $75(2)$ \\
\hline $\mathrm{O}(11)$ & $326(10)$ & $4922(12)$ & $2483(2)$ & $108(3)$ \\
\hline $\mathrm{O}(12)$ & $-840(12)$ & $7633(12)$ & $1643(3)$ & $118(3)$ \\
\hline $\mathrm{O}(13)$ & $-443(8)$ & $6315(8)$ & $442(2)$ & $77(2)$ \\
\hline $\mathrm{O}(14)$ & $3034(11)$ & $10256(9)$ & $1366(3)$ & $93(2)$ \\
\hline
\end{tabular}

\section{Results and discussion}

\subsection{Synthesis}

$\mathrm{Cp}_{2} \mathrm{Mo}_{2}(\mathrm{CO})_{4}\left(\mu-\eta^{2}-\mathrm{C}_{14} \mathrm{H}_{20}\right)$ (1) was previously prepared by treating cyclotetradeca-1,8-diyne $\left(\mathrm{C}_{14} \mathrm{H}_{20}\right)$ with the Mo-Mo triple-bonded species $\mathrm{Cp}_{2} \mathrm{Mo}_{2}(\mathrm{CO})_{4}$ [7]. The reactions of $\mathbf{1}$ and iron group carbonyl clusters are summarized in Scheme 1. Compound 1 reacts with $\mathrm{Fe}_{3}(\mathrm{CO})_{12}$ in refluxing hexane to produce the bis-alkyne complex $\left[\mathrm{Cp}_{2} \mathrm{Mo}_{2}(\mathrm{CO})_{4}\right]\left(\mu, \mu_{3}-\eta^{2}, \eta^{2}-\mathrm{C}_{14} \mathrm{H}_{20}\right)\left[\mathrm{Fe}_{3}(\mathrm{CO})_{9}\right]$ (2) in $33 \%$ yield, while the similar reaction with $\mathrm{Ru}_{3}(\mathrm{CO})_{12}$ requires a higher temperature (refluxing heptane) to afford the alkyne-allyl complex $\left[\mathrm{Cp}_{2} \mathrm{Mo}_{2}-\right.$ $\left.(\mathrm{CO})_{4}\right]\left(\mu, \mu_{3}-\eta^{2}, \eta^{3}-\mathrm{C}_{14} \mathrm{H}_{19}\right)\left[(\mu-\mathrm{H}) \mathrm{Ru}_{3}(\mathrm{CO})_{9}\right] \quad$ (3-allyl; $52 \%$ ). In contrast, there is no reaction between $\mathrm{Os}_{3}(\mathrm{CO})_{12}$ and 1 up to $110^{\circ} \mathrm{C}$ (refluxing toluene) but slow decomposition of the reactants occurs. This difference in reactivity may be attributed to stronger Os- $\mathrm{CO}$ bonds in comparison with the $\mathrm{Ru}-\mathrm{CO}$ and $\mathrm{Fe}-\mathrm{CO}$ bonds [15]. Thus, compound 1 reacts with the more labile acetonitrile-substituted complex $\mathrm{Os}_{3}(\mathrm{CO})_{10^{-}}$ $(\mathrm{NCMe})_{2}$ at $70^{\circ} \mathrm{C}$ to afford the bis-alkyne complex $\left[\mathrm{Cp}_{2} \mathrm{Mo}_{2}(\mathrm{CO})_{4}\right]\left(\mu, \mu_{3}-\eta^{2}, \eta^{2}-\mathrm{C}_{14} \mathrm{H}_{20}\right)\left[\mathrm{Os}_{3}(\mathrm{CO})_{10}\right]$ (4) in good yield $(90 \%)$. An alternative method to prepare 2 and 4 is from $\mathrm{Cp}_{2} \mathrm{Mo}_{2}(\mathrm{CO})_{4}$ reacting with $\mathrm{Fe}_{3}(\mathrm{CO})_{9}\left(\mu_{3}-\right.$ $\left.\eta^{2}-\mathrm{C}_{14} \mathrm{H}_{20}\right)$ and $\mathrm{Os}_{3}(\mathrm{CO})_{10}\left(\mu_{3}-\eta^{2}-\mathrm{C}_{14} \mathrm{H}_{20}\right)$ [6], respectively.

It is of interest that $\mathrm{Ru}_{3}(\mathrm{CO})_{10}(\mathrm{NCMe})_{2}$ reacts with $\mathbf{1}$ at $25^{\circ} \mathrm{C}$ to produce the alkyne-allenyl complex $\left[\mathrm{Cp}_{2} \mathrm{Mo}_{2}(\mathrm{CO})_{4}\right]\left(\mu, \mu_{3}-\eta^{2}, \eta^{3}-\mathrm{C}_{14} \mathrm{H}_{19}\right)\left[(\mu-\mathrm{H}) \mathrm{Ru}_{3}(\mathrm{CO})_{9}\right]$ allenyl; $88 \%)$. Presumably, the bis-alkyne intermediate $\left[\mathrm{Cp}_{2} \mathrm{Mo}_{2}(\mathrm{CO})_{4}\right]\left(\mu, \mu_{3}-\eta^{2}, \eta^{2}-\mathrm{C}_{14} \mathrm{H}_{20}\right)\left[\mathrm{Ru}_{3}(\mathrm{CO})_{10}\right]$, analogous to $\mathbf{4}$, is initially afforded, which then undergoes a facile $\beta-\mathrm{C}-\mathrm{H}$ activation of the alkyne ligand and a $\mathrm{CO}$ loss to give 3-allenyl. Tunik and co-workers [16] previously reported the reaction of $\mathrm{Ru}_{3}(\mathrm{CO})_{12}$ and hexa-2,4diyne-1,6-diol to lead to the alkyne complex $\mathrm{Ru}_{3}(\mathrm{CO})_{10}\left(\mu_{3}-\eta^{2}-\mathrm{HOCH}_{2} \mathrm{C}_{2} \mathrm{C}_{2} \mathrm{CH}_{2} \mathrm{OH}\right)$ in $14 \%$, while the transformation from a coordinated alkyne to a hydrido-allenyl moiety has been established in some triosmium systems [6,17-19]. Thermolysis of 3-allenyl at $70^{\circ} \mathrm{C}$ results in a hydrogen migration to afford 3-allyl. Though the last isomerization was known for $(\mu-\mathrm{H}) \mathrm{Ru}_{3}(\mathrm{CO})_{9}\left(\mu_{3}-\eta^{2}-\mathrm{EtC}=\mathrm{C}=\mathrm{C}(\mathrm{Me}) \mathrm{H}\right)$ to $(\mu-\mathrm{H}) \mathrm{Ru}_{3}-$ $(\mathrm{CO})_{9}\left(\mu_{3}-\eta^{2}\right.$-EtCCHCMe $)$ [20], it remains unclear whether the allyl-hydrogen is derived from a direct 1,2-hydrogen shift of the allenyl moiety, or from migration of the bridging hydride concomitant with an allenyl $\mathrm{C}-\mathrm{H}$ activation on the cluster. It is noteworthy that the reversed transformation was established for the hydrido allyl complex $(\mu-\mathrm{H}) \mathrm{Ru}_{3}(\mathrm{CO})_{9}\left(\mu_{3}-\eta^{3}-\mathrm{HCCH}-\right.$ $\mathrm{COMe})$ to the dihydrido alkyne complex ( $\mu$ $\mathrm{H})_{2} \mathrm{Ru}_{3}(\mathrm{CO})_{9}\left(\mu_{3}-\eta^{2}-\mathrm{MeCCOMe}\right)$ upon hydrogenation, presumably through an allenyl intermediate [21]. 


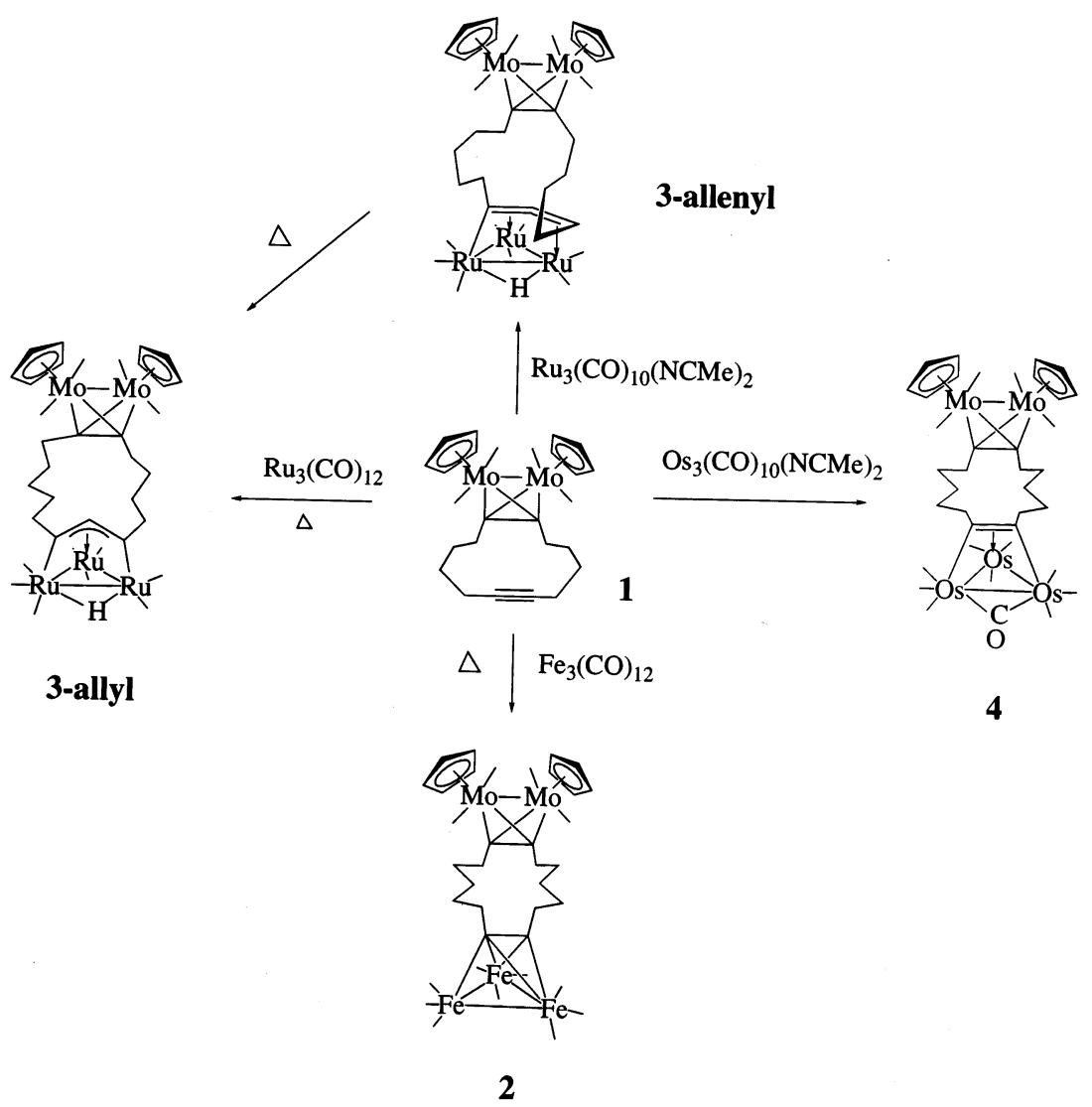

Scheme 1.

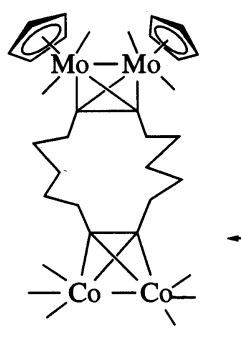

5

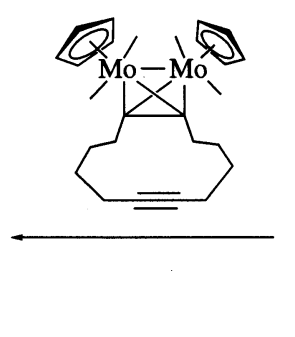

$\mathrm{Co}_{2}(\mathrm{CO})_{8}$

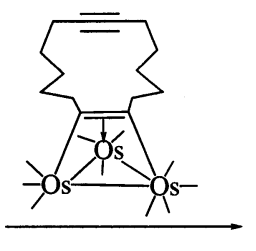

6

Scheme 2 .

The heterometallic bis-alkyne clusters involving a $\mathrm{Co}_{2}$ unit are prepared similarly at ambient temperature. The reaction of $\mathbf{1}$ with $\mathrm{Co}_{2}(\mathrm{CO})_{8}$ produces $\left[\mathrm{Cp}_{2} \mathrm{Mo}_{2}(\mathrm{CO})_{4}\right]\left(\mu, \mu-\eta^{2}, \eta^{2}-\mathrm{C}_{14} \mathrm{H}_{20}\right)\left[\mathrm{Co}_{2}(\mathrm{CO})_{6}\right] \quad(\mathbf{5} ; 95 \%)$, and of $\mathrm{Os}_{3}(\mathrm{CO})_{10}\left(\mu_{3}-\eta^{2}-\mathrm{C}_{14} \mathrm{H}_{20}\right)$ with $\mathrm{Co}_{2}(\mathrm{CO})_{8}$ affords $\left[\mathrm{Os}_{3}(\mathrm{CO})_{10}\right]\left(\mu_{3}, \mu-\eta^{2}, \eta^{2}-\mathrm{C}_{14} \mathrm{H}_{20}\right)\left[\mathrm{Co}_{2}(\mathrm{CO})_{6}\right] \quad(\mathbf{6} ; \quad 88 \%)$ (Scheme 2).

\subsection{Spectroscopic characterization of new complexes}

Compounds 2-6 form air-stable crystalline solids and have been characterized by mass, IR and NMR spectroscopy. The FAB mass spectra of these com- plexes display their molecular ion peaks at $m / z=1046$, 1184, 1482, 912 and 1330 for 2, 3 (allyl and allenyl), 4, 5 and 6, respectively, for the ${ }^{56} \mathrm{Fe},{ }^{98} \mathrm{Mo},{ }^{102} \mathrm{Ru}$, and ${ }^{192} \mathrm{Os}$ isotopes. The isotopic distribution of the envelope surrounding the molecular ions match that expected for these compounds, and there is good agreement between the calculated mass distribution and the observed mass spectrum, such as Fig. 1 for 3-allyl.

The IR spectrum in the carbonyl region of $\mathbf{2}$ is apparently that of $\mathrm{Cp}_{2} \mathrm{Mo}_{2}(\mathrm{CO})_{4}\left(\mu-\eta^{2}-\mathrm{C}_{14} \mathrm{H}_{20}\right)$ (1) plus $\mathrm{Fe}_{3}(\mathrm{CO})_{9}\left(\mu_{3}-\eta^{2}-\mathrm{C}_{14} \mathrm{H}_{20}\right)$, indicating small interactions between the two metal systems in $\mathbf{2}$ spanned by a 14-membered carbocyclic ring. Thus, the coordination 
about the triiron parts of $\mathbf{2}$ should closely resemble $\mathrm{Fe}_{3}(\mathrm{CO})_{9}\left(\mu_{3}-\eta^{2}-\mathrm{C}_{2} \mathrm{R}_{2}\right)(\mathrm{R}=\mathrm{Et}$ [22] and $\mathrm{Ph}$ [23] $)$ and $\mathrm{Fe}_{3}(\mathrm{CO})_{9}\left(\mu_{3}-\eta^{2}-\mathrm{C}_{14} \mathrm{H}_{20}\right)[6]$, in which the alkyne triple bond is disposed above the $\mathrm{Fe}_{3}$ plane and perpendicular to one of the $\mathrm{Fe}-\mathrm{Fe}$ edge, with one acetylenic carbon in a $\mu_{3}$ mode and the other in a $\mu_{2}$ mode. The ${ }^{13} \mathrm{C}\left\{{ }^{1} \mathrm{H}\right\}$ NMR spectrum of $\mathbf{2}$ includes three coordinated alkyne carbon resonances at 207.7, 109.4 (to $\mathrm{Fe}_{3}$ ) and 113.6 (to $\mathrm{Mo}_{2}$ ) ppm, consistent with the proposed structure.

Compounds 3-allyl and 3-allenyl form brown-red crystals. They differ only in the bonding and the arrangement of the organic ligand with respect to the $\mathrm{Ru}_{3}$ cluster. The ${ }^{1} \mathrm{H}-\mathrm{NMR}$ spectrum of 3-allyl presents the allyl proton resonance at $7.80 \mathrm{ppm}$ and the bridging hydride resonance at $-19.70 \mathrm{ppm}$, while 3-allenyl shows the allenyl proton resonance at $3.55 \mathrm{ppm}$ and the bridging hydride resonance at $-20.57 \mathrm{ppm}$.

Compound 4 forms brown crystals. Its NMR spectrum displays five sets of ${ }^{1} \mathrm{H}$ resonances in the range $2.92-1.30 \mathrm{ppm}$ and seven ${ }^{13} \mathrm{C}$ resonance signals at 148.4 $(\mathrm{C} \equiv \mathrm{C}-\mathrm{Os}), 111.5(\mathrm{C} \equiv \mathrm{C}-\mathrm{Mo}), 91.0,49.2,37.8,30.8$ and $28.3 \mathrm{ppm}$ for the $\mathrm{C}_{14} \mathrm{H}_{20}$ ligand. The ${ }^{1} \mathrm{H}$ - and ${ }^{13} \mathrm{C}-\mathrm{NMR}$ data are consistent with a molecule of idealized $C_{s}$ symmetry in solution. There is no clear indication for
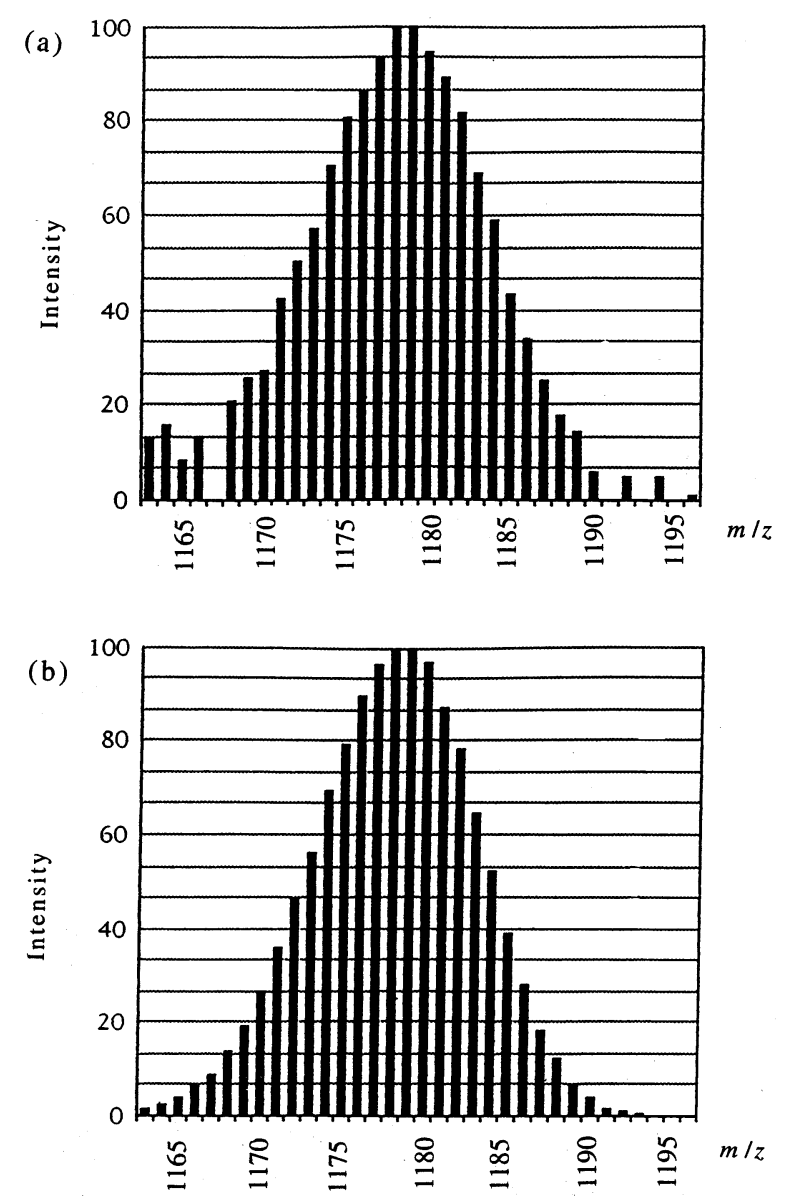

Fig. 1. The observed (a) and calculated (b) isotopic distribution for the molecular ion peaks of 3-allyl. the presence of bridging carbonyl associated with the $\mathrm{Os}_{3}$ cluster, as parts of Mo-CO absorptions appearing in the region $1900-1800 \mathrm{~cm}^{-1}$. A single-crystal X-ray diffraction study of $\mathbf{4}$ was therefore conducted.

The cobalt atom containing clusters 5 and $\mathbf{6}$ form brown crystals. Their IR spectra include a CO absorption pattern in close agreement with that of $\mathrm{Co}_{2}(\mathrm{CO})_{6}\left(\mu-\eta^{2}-\mathrm{RCCR}^{\prime}\right)[24,25]$. Thus, the two alkyne ligands of $\mathbf{5}$ are each likely to be coordinated to $\mathrm{Mo}_{2}$ and $\mathrm{Co}_{2}$ units in a perpendicular bonding mode to form two tetrahedral $\mathrm{C}_{2} \mathrm{M}_{2}$ cores. The IR and NMR data are consistent with a symmetric structure for $\mathbf{5}$. The solution IR spectrum of $\mathbf{6}$ present a very weak and broad absorption at $1845 \mathrm{~cm}^{-1}$ for bridging carbonyl. We note that a weak absorption at $1850 \mathrm{~cm}^{-1}$ was also recorded for the parent complex $\mathrm{Os}_{3}(\mathrm{CO})_{10}\left(\mu_{3}-\eta^{2}\right.$ $\mathrm{C}_{14} \mathrm{H}_{20}$ ) [6]. The ${ }^{13} \mathrm{C}\left\{{ }^{1} \mathrm{H}\right\}$-NMR spectrum of 6 shows two coordinated alkyne carbon resonances at 148.3 (to $\mathrm{Os}_{3}$ ) and 98.2 (to $\left.\mathrm{Co}_{2}\right) \mathrm{ppm}$, and five resonances ranging from 48.2 to $28.0 \mathrm{ppm}$ for the methylene carbons, suggesting a $C_{s}$ symmetry for the molecule.

\subsection{Crystal structure of 3-allyl}

Crystals of $\left[\mathrm{Cp}_{2} \mathrm{Mo}_{2}(\mathrm{CO})_{4}\right]\left(\mu, \mu_{3}-\eta^{2}, \eta^{3}-\mathrm{C}_{14} \mathrm{H}_{19}\right)[(\mu-$ $\mathrm{H}) \mathrm{Ru}_{3}(\mathrm{CO})_{9}$ ] (3-allyl) contain an ordered array of discrete monomeric molecular units which are mutually separated by normal van der Waals distances. The ORTEP diagram is shown in Fig. 2. Selected bond distances and bond angles are given in Table 4 .

The trimetal parts are based on a triangular array of ruthenium atoms in which the $\mathrm{Ru}(1)-\mathrm{Ru}(3)$ distance of 2.953(1) $\AA$ is significantly longer than the other two intermetallic distances (i.e. an $\mathrm{Ru}(1)-\mathrm{Ru}(2)$ distance of 2.787(1) and an $\mathrm{Ru}(2)-\mathrm{Ru}(3)$ distance of 2.785(1) $\AA$ ). The hydride atom is not located, but the distribution of the carbonyl groups and the $\mathrm{Ru}-\mathrm{Ru}$ lengths indicate that it probably spans the $\mathrm{Ru}(1)-\mathrm{Ru}(3)$ edge. Each ruthenium atom is associated with three terminal carbonyl ligands. The $\mathrm{Ru}-\mathrm{CO}$ distances range from 1.88(1) $(\mathrm{Ru}(3)-\mathrm{C}(9))$ to $1.97(1) \AA(\mathrm{Ru}(3)-\mathrm{C}(7))$, while the $\mathrm{Ru}-\mathrm{C}-\mathrm{O}$ angles are in the range $173(1)-178(1)^{\circ}$.

The neutral allyl ligand $\mathrm{C}(14)-\mathrm{C}(16)$ donates five electrons to the cluster and can be described as forming $\sigma$ bonds to $\mathrm{Ru}(1)$ and $\mathrm{Ru}(3)$, with $\mathrm{C}(14)-\mathrm{Ru}(1)=$ 2.090(9) and $\mathrm{C}(16)-\mathrm{Ru}(3)=2.068(9) \AA$, and an $\eta^{3}-\pi$ bond to $\mathrm{Ru}(2)$, where the $\mathrm{Ru}(2)-\mathrm{C}$ distance to the internal $\mathrm{C}(15)$ atom $(2.248(8) \AA$ ) is slightly shorter than that to the external $\mathrm{C}(14)(2.277(9) \AA)$ and $\mathrm{C}(16)$ (2.270(9) $\AA$ ) atoms. The $\mathrm{Ru}(1), \mathrm{C}(14)-\mathrm{C}(16)$, and $\mathrm{Ru}(3)$ atoms are coplanar to within $0.03 \AA$, and the dihedral angle between this plane and the triruthenium plane is $50.2^{\circ}$. The allyl $\mathrm{C}(14)-\mathrm{C}(15)$ and $\mathrm{C}(15)-\mathrm{C}(16)$ distances of 1.41(1) and 1.40(1) $\AA$ are characteristic of partial carbon-carbon double bonds. 


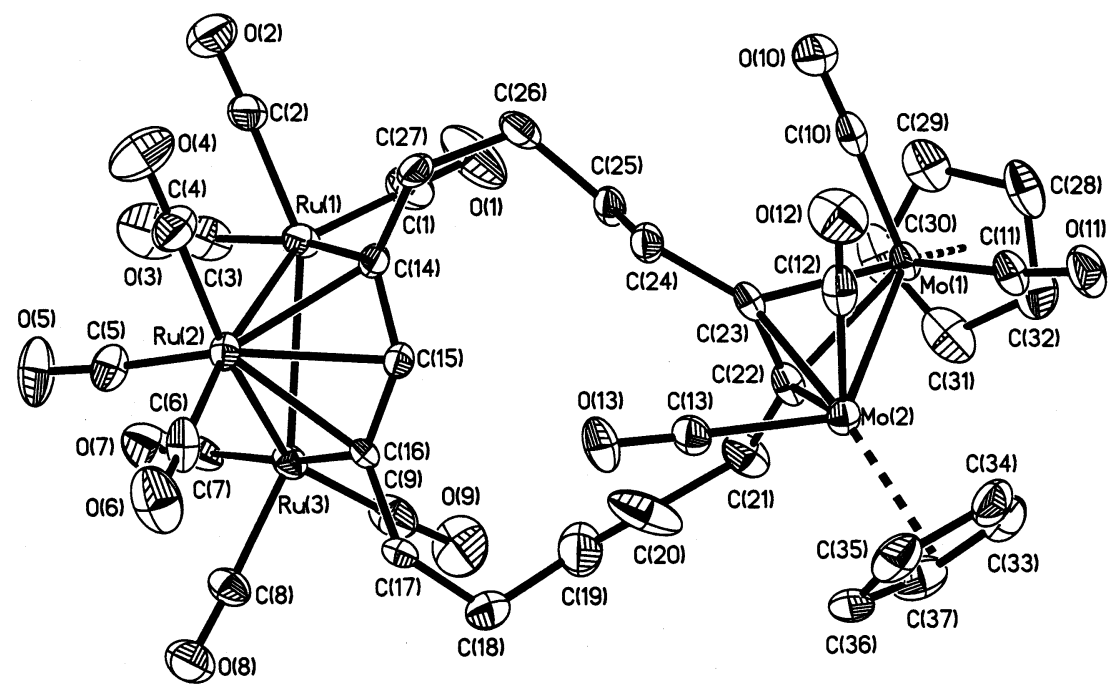

Fig. 2. Molecular structure of $\left[\mathrm{Cp}_{2} \mathrm{Mo}_{2}(\mathrm{CO})_{4}\right]\left(\mu, \mu_{3}-\eta^{2}, \eta^{3}-\mathrm{C}_{14} \mathrm{H}_{19}\right)\left[(\mu-\mathrm{H}) \mathrm{Ru} u_{3}(\mathrm{CO})_{9}\right]$ (3-allyl). The hydrogen atoms have been artificially omitted for clarity.

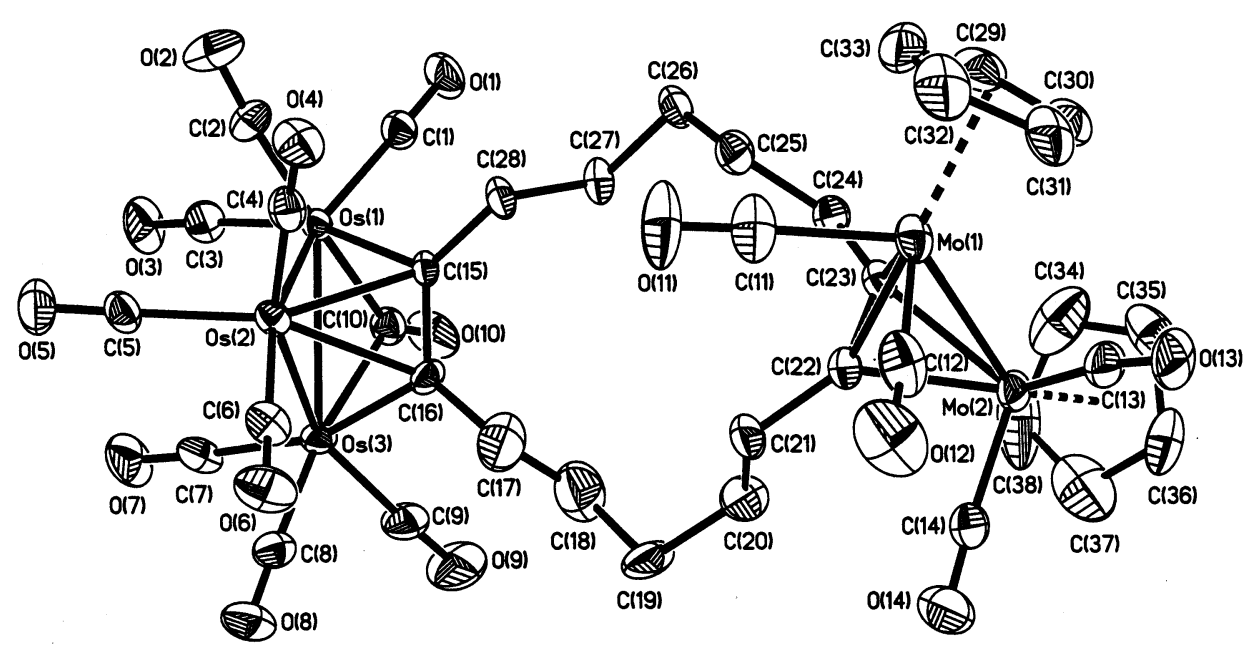

Fig. 3. Molecular structure of $\left[\mathrm{Cp}_{2} \mathrm{Mo}_{2}(\mathrm{CO})_{4}\right]\left(\mu, \mu_{3}-\eta^{2}, \eta^{2}-\mathrm{C}_{14} \mathrm{H}_{20}\right)\left[\mathrm{Os}_{3}(\mathrm{CO})_{10}\right](4)$. The hydrogen atoms have been artificially omitted for clarity.

The alkyne $C(22)-C(23)$ unit apparently donates its four $\pi$-electrons to the dimolybdenum system in a $\perp$-bonding mode to give rise to a quasi-tetrahedral core, while the $\mathrm{C}(23)-\mathrm{Mo}(2)$ length $(2.275(9) \AA)$ is significantly longer than the other three lengths: $\mathrm{C}(23)-\mathrm{Mo}(1)=2.194(8), \quad \mathrm{C}(22)-\mathrm{Mo}(1)=2.176(9)$ and $\mathrm{C}(22)-\mathrm{Mo}(2)=2.168(9) \AA$. The $\mathrm{C}(22)-\mathrm{C}(23)$ distance is 1.34(1) $\AA$, ca. $0.15 \AA$ longer than that in free alkyne form. Application of the 18 -electron rule requires a Mo-Mo single bond for 3-allyl. The $\operatorname{Mo}(1)-\operatorname{Mo}(2)$ distance of 2.979(1) $\AA$ is comparable with the analogous alkyne complexes $\mathrm{Cp}_{2} \mathrm{Mo}_{2}(\mathrm{CO})_{4}(\mathrm{RCCR})$ ( $\mathrm{R}=\mathrm{H}$, Et and $\mathrm{Ph}[26])$ but is between a Mo-Mo single bond length measured for $\mathrm{Cp}_{2} \mathrm{Mo}_{2}(\mathrm{CO})_{6}(3.235 \AA$ ) [27] and a Mo-Mo triple bond length for $\mathrm{Cp}_{2} \mathrm{Mo}_{2}(\mathrm{CO})_{4}$ (2.448 ̊) [28].
The $\mathrm{Cp}$ and carbonyl ligands connected to the $\mathrm{C}_{2} \mathrm{Mo}_{2}$ core are staggered to reduce steric repulsions. The two cyclopentadienyl groups are each bonded to a molybdenum atom asymmetrically, with the $\mathrm{Mo}-\mathrm{C}$ distances ranging from 2.30(1) to 2.39(1) $\AA$. The $\mathrm{Mo}(1)$ and $\mathrm{Mo}(2)$ atoms are each linked to two terminal carbonyls with the Mo-C lengths ranging from 1.94(1) to $1.97(1) \AA$. The $\mathrm{Mo}(1)-\mathrm{C}(10)-\mathrm{O}(10), \mathrm{Mo}(2)-\mathrm{C}(12)-$ $\mathrm{O}(12)$ and $\mathrm{Mo}(2)-\mathrm{C}(13)-\mathrm{O}(13)$ angles are 176.8(9), 177.1(9) and $178.6(9)^{\circ}$, respectively, while the $\mathrm{Mo}(1)$ $\mathrm{C}(11)-\mathrm{O}(11)$ angle is $169.5(8)^{\circ}$. The last Mo-C-O angle deviates slightly but significantly from linearity and shows weak bonding with the adjacent Mo atom, such as $\operatorname{Mo}(2) \cdots \mathrm{C}(11)=2.836 \AA$, clearly indicating a semibridging interaction [29]. 
Selected bond distances $(\AA)$ and bond angles $\left({ }^{\circ}\right)$ for 3-allyl and 4

\begin{tabular}{|c|c|c|}
\hline & 3-allyl $(M=R u)$ & $4(\mathrm{M}=\mathrm{Os})$ \\
\hline \multicolumn{3}{|l|}{ Bond distances } \\
\hline $\mathrm{M}(1)-\mathrm{M}(2)$ & $2.787(1)$ & $2.7614(3)$ \\
\hline $\mathrm{M}(1)-\mathrm{M}(3)$ & $2.953(1)$ & $2.8368(4)$ \\
\hline $\mathbf{M}(2)-\mathbf{M}(3)$ & $2.785(1)$ & $2.7620(4)$ \\
\hline $\operatorname{Mo}(1)-\operatorname{Mo}(2)$ & $2.979(1)$ & $2.9555(9)$ \\
\hline $\mathrm{M}(1)-\mathrm{C}(1)$ & $1.92(1)$ & $1.923(7)$ \\
\hline $\mathrm{M}(1)-\mathrm{C}(2)$ & $1.90(1)$ & $1.933(8)$ \\
\hline $\mathrm{M}(1)-\mathrm{C}(3)$ & $1.95(1)$ & $1.959(8)$ \\
\hline$M(2)-C(4)$ & $1.92(1)$ & $1.914(8)$ \\
\hline$M(2)-C(5)$ & $1.90(1)$ & $1.933(8)$ \\
\hline$M(2)-C(6)$ & $1.91(1)$ & $1.898(8)$ \\
\hline$M(3)-C(7)$ & $1.97(1)$ & $1.956(9)$ \\
\hline $\mathrm{M}(3)-\mathrm{C}(8)$ & $1.91(1)$ & $1.905(8)$ \\
\hline $\mathrm{M}(3)-\mathrm{C}(9)$ & $1.88(1)$ & $1.908(9)$ \\
\hline $\mathrm{M}(1)-\mathrm{C}(10)$ & & $2.083(7)$ \\
\hline $\mathrm{M}(3)-\mathrm{C}(10)$ & & $2.291(7)$ \\
\hline $\mathrm{Mo}(1)-\mathrm{C}(10)$ & $1.94(1)$ & \\
\hline $\mathrm{Mo}(1)-\mathrm{C}(11)$ & $1.95(1)$ & $1.98(1)$ \\
\hline $\mathrm{Mo}(1)-\mathrm{C}(12)$ & & $1.97(1)$ \\
\hline $\mathrm{Mo}(2)-\mathrm{C}(12)$ & $1.97(1)$ & \\
\hline $\operatorname{Mo}(2)-C(13)$ & $1.96(1)$ & $1.945(8)$ \\
\hline $\mathrm{Mo}(2)-\mathrm{C}(14)$ & & $1.95(1)$ \\
\hline $\mathrm{M}(1)-\mathrm{C}(14)$ & $2.090(9)$ & \\
\hline $\mathrm{M}(2)-\mathrm{C}(14)$ & $2.277(9)$ & \\
\hline $\mathrm{M}(1)-\mathrm{C}(15)$ & & $2.133(6)$ \\
\hline $\mathrm{M}(2)-\mathrm{C}(15)$ & $2.248(8)$ & $2.261(6)$ \\
\hline $\mathrm{M}(2)-\mathrm{C}(16)$ & $2.270(9)$ & $2.288(7)$ \\
\hline $\mathrm{M}(3)-\mathrm{C}(16)$ & $2.068(9)$ & $2.119(7)$ \\
\hline $\mathrm{Mo}(1)-\mathrm{C}(22)$ & $2.176(9)$ & $2.289(7)$ \\
\hline $\mathrm{Mo}(2)-\mathrm{C}(22)$ & $2.168(9)$ & $2.220(7)$ \\
\hline $\mathrm{Mo}(1)-\mathrm{C}(23)$ & $2.194(8)$ & $2.165(7)$ \\
\hline $\mathrm{Mo}(2)-\mathrm{C}(23)$ & $2.275(9)$ & $2.188(7)$ \\
\hline $\mathrm{Mo}(1)-\mathrm{C}(28)$ & $2.32(1)$ & \\
\hline $\mathrm{Mo}(1)-\mathrm{C}(29)$ & $2.33(1)$ & $2.37(1)$ \\
\hline $\mathrm{Mo}(1)-\mathrm{C}(30)$ & $2.36(1)$ & $2.370(9)$ \\
\hline $\mathrm{Mo}(1)-\mathrm{C}(31)$ & $2.38(1)$ & $2.332(8)$ \\
\hline $\mathrm{Mo}(1)-\mathrm{C}(32)$ & $2.36(1)$ & $2.305(9)$ \\
\hline $\mathrm{Mo}(1)-\mathrm{C}(33)$ & & $2.32(1)$ \\
\hline $\operatorname{Mo}(2)-C(33)$ & $2.39(1)$ & \\
\hline $\operatorname{Mo}(2)-\mathrm{C}(34)$ & $2.32(1)$ & $2.36(1)$ \\
\hline $\operatorname{Mo}(2)-\mathrm{C}(35)$ & $2.30(1)$ & $2.35(1)$ \\
\hline $\mathrm{Mo}(2)-\mathrm{C}(36)$ & $2.32(1)$ & $2.32(1)$ \\
\hline $\mathrm{Mo}(2)-\mathrm{C}(37)$ & $2.36(1)$ & $2.32(1)$ \\
\hline $\operatorname{Mo}(2)-C(38)$ & & $2.32(1)$ \\
\hline$C(14)-(15)$ & $1.41(1)$ & \\
\hline$C(15)-(16)$ & $1.40(1)$ & $1.412(9)$ \\
\hline $\mathrm{C}(22)-\mathrm{C}(23)$ & $1.34(1)$ & $1.35(1)$ \\
\hline $\mathrm{C}(10)-\mathrm{O}(10)$ & $1.17(1)$ & $1.132(8)$ \\
\hline \multicolumn{3}{|l|}{ Bond angles } \\
\hline $\mathrm{M}(1)-\mathrm{M}(2)-\mathrm{M}(3)$ & $64.00(3)$ & $61.807(9)$ \\
\hline $\mathrm{M}(1)-\mathrm{M}(3)-\mathrm{M}(2)$ & $58.04(3)$ & $59.086(9)$ \\
\hline $\mathrm{M}(2)-\mathrm{M}(1)-\mathrm{M}(3)$ & $57.96(3)$ & $59.106(9)$ \\
\hline $\mathrm{M}(1)-\mathrm{C}(1)-\mathrm{O}(1)$ & $178(1)$ & $178.4(7)$ \\
\hline $\mathrm{M}(1)-\mathrm{C}(2)-\mathrm{O}(2)$ & $177.4(9)$ & 171.7(7) \\
\hline $\mathrm{M}(1)-\mathrm{C}(3)-\mathrm{O}(3)$ & $175(1)$ & $175.5(8)$ \\
\hline $\mathrm{M}(2)-\mathrm{C}(4)-\mathrm{O}(4)$ & $178(1)$ & $178.4(7)$ \\
\hline $\mathrm{M}(2)-\mathrm{C}(5)-\mathrm{O}(5)$ & $173(1)$ & $176.7(7)$ \\
\hline $\mathrm{M}(2)-\mathrm{C}(6)-\mathrm{O}(6)$ & $178(1)$ & $179.7(8)$ \\
\hline $\mathrm{M}(3)-\mathrm{C}(7)-\mathrm{O}(7)$ & $178(1)$ & $175.6(8)$ \\
\hline $\mathrm{M}(3)-\mathrm{C}(8)-\mathrm{O}(8)$ & $177.4(9)$ & $176.7(7)$ \\
\hline $\mathrm{M}(3)-\mathrm{C}(9)-\mathrm{O}(9)$ & $175(1)$ & $177.5(9)$ \\
\hline
\end{tabular}

Table 4 (Continued)

$\begin{array}{lrr}\mathrm{M}(1)-\mathrm{C}(10)-\mathrm{O}(10) & & 145.3(7) \\ \mathrm{M}(3)-\mathrm{C}(10)-\mathrm{O}(10) & & 134.0(6) \\ \mathrm{M}(1)-\mathrm{C}(10)-\mathrm{M}(3) & & 80.7(2) \\ \mathrm{Mo}(1)-\mathrm{C}(10)-\mathrm{O}(10) & 176.8(9) & 178(1) \\ \mathrm{Mo}(1)-\mathrm{C}(11)-\mathrm{O}(11) & 169.5(8) & 177(1) \\ \mathrm{Mo}(1)-\mathrm{C}(12)-\mathrm{O}(12) & & \\ \mathrm{Mo}(2)-\mathrm{C}(12)-\mathrm{O}(12) & 177.1(9) & 168.4(8) \\ \mathrm{Mo}(2)-\mathrm{C}(13)-\mathrm{O}(13) & 178.6(9) & 177.6(8) \\ \mathrm{Mo}(2)-\mathrm{C}(14)-\mathrm{O}(14) & & \\ \mathrm{M}(1)-\mathrm{C}(14)-\mathrm{C}(15) & 122.0(7) & \\ \mathrm{M}(3)-\mathrm{C}(16)-\mathrm{C}(15) & 124.3(7) & \\ \mathrm{C}(14)-\mathrm{C}(15)-\mathrm{C}(16) & 125.5(8) & 109.4(4) \\ \mathrm{C}(15)-\mathrm{C}(14)-\mathrm{C}(27) & 114.9(8) & 109.8(4) \\ \mathrm{C}(15)-\mathrm{C}(16)-\mathrm{C}(17) & 113.9(8) & 124.3(7) \\ \mathrm{M}(1)-\mathrm{C}(15)-\mathrm{C}(16) & & 123.6(6) \\ \mathrm{M}(3)-\mathrm{C}(16)-\mathrm{C}(15) & & 50.1(2) \\ \mathrm{C}(15)-\mathrm{C}(16)-\mathrm{C}(17) & & 46.9(2) \\ \mathrm{C}(16)-\mathrm{C}(15)-\mathrm{C}(28) & & 47.5(2) \\ \mathrm{Mo}(1)-\mathrm{Mo}(2)-\mathrm{C}(22) & 46.8(2) & 47.6(2) \\ \mathrm{Mo}(1)-\mathrm{Mo}(2)-\mathrm{C}(23) & 47.0(2) & 77.4(4) \\ \mathrm{Mo}(2)-\mathrm{Mo}(1)-\mathrm{C}(22) & 46.6(2) & 72.5(4) \\ \mathrm{Mo}(2)-\mathrm{Mo}(1)-\mathrm{C}(23) & 49.4(2) & 67.4(4) \\ \mathrm{C}(22)-\mathrm{C}(23)-\mathrm{Mo}(1) & 71.4(5) & 135.5(7) \\ \mathrm{C}(22)-\mathrm{C}(23)-\mathrm{Mo}(2) & 68.1(5) & 132.2(6) \\ \mathrm{C}(23)-\mathrm{C}(22)-\mathrm{Mo}(1) & 72.8(6) & 76.8(6) \\ \mathrm{C}(23)-\mathrm{C}(22)-\mathrm{Mo}(2) & 134.6(9) & \\ \mathrm{C}(21)-\mathrm{C}(22)-\mathrm{C}(23) & 137.6(9) & \\ \mathrm{C}(22)-\mathrm{C}(23)-\mathrm{C}(24) & & \\ \end{array}$

\subsection{Crystal structure of $\mathbf{4}$}

The ORTEP drawing of $\left[\mathrm{Cp}_{2} \mathrm{Mo}_{2}(\mathrm{CO})_{4}\right]\left(\mu, \mu_{3}-\eta^{2}, \eta^{2}-\right.$ $\left.\mathrm{C}_{14} \mathrm{H}_{20}\right)\left[\mathrm{Os}_{3}(\mathrm{CO})_{10}\right]$ (4) (Fig. 3) shows that the two alkyne triple bonds of the $\mathrm{C}_{14} \mathrm{H}_{20}$ ligand are each bonded to a $\mathrm{Mo}_{2}$ and an $\mathrm{Os}_{3}$ moieties. Important interatomic distances and intramolecular angles are collected in Table 4.

The trimetal parts consist of an isosceles triangular cluster of three $\mathrm{Os}(\mathrm{CO})_{3}$ groups with a triply bridging alkyne and a doubly bridging carbonyl ligands. The dibridged Os(1)-Os(3) distance of 2.8368(4) $\AA$ is significantly longer than the other two intermetallic distances, i.e. $\operatorname{Os}(1)-\mathrm{Os}(2)=2.7614(3)$ and $\mathrm{Os}(2)-\mathrm{Os}(3)=$ $2.7620(4) \AA$. A similar difference was found in $\mathrm{Os}_{3}(\mathrm{CO})_{9}(\mu-\mathrm{CO})\left(\mu_{3}-\eta^{2}-\mathrm{C}_{2} \mathrm{Et}_{2}\right) \quad[30], \quad \mathrm{Os}_{3}(\mathrm{CO})_{9}(\mu-$ $\mathrm{C}(\mathrm{OMe}) \mathrm{Ph})\left(\mu_{3}-\eta^{2}-\mathrm{C}_{2} \mathrm{Ph}_{2}\right)[31]$ and $\mathrm{Os}_{3}(\mathrm{CO})_{9}\left(\mu-\mathrm{CH}_{2}\right)\left(\mu_{3}-\right.$ $\eta^{2}-\mathrm{C}_{2} \mathrm{Ph}_{2}$ ) [32]. However, the Os-Os distances in the analogous double cluster $\left[\mathrm{Os}_{3}(\mathrm{CO})_{10}\right]_{2}\left(\mu_{3}, \mu_{3}-\eta^{2}, \eta^{2}-\right.$ $\mathrm{C}_{14} \mathrm{H}_{20}$ ), which contains no bridging $\mathrm{CO}$, show substantial variation, being $2.7087(6), 2.8570(5)$ and $2.8776(5)$ $\AA$ [6].

The alkyne $\mathrm{C}(15)-\mathrm{C}(16)$ unit is coordinated to three osmium atoms, formally forming a $\pi$ bond to Os(2), with $\mathrm{Os}(2)-\mathrm{C}(15)=2.261(6)$ and $\mathrm{Os}(2)-\mathrm{C}(16)=$ 2.288(7), and $\sigma$ bonds to Os(1) and Os(3) atoms, with $\operatorname{Os}(1)-\mathrm{C}(15)=2.133(6)$ and $\operatorname{Os}(3)-\mathrm{C}(16)=2.119(7) \AA$. The $\mathrm{C}(15), \mathrm{C}(16), \mathrm{Os}(3)$, and $\mathrm{Os}(1)$ atoms are coplanar, 
and the dihedral angle between this plane and the triosmium plane is $58.56^{\circ}$. The Os(1)-Os(3) edge is also bridged by a carbonyl asymmetrically, such that the Os(1)-C(10) and Os(3)-C(10) distances are 2.083(7) and 2.291(7) $\AA$, and the $\mathrm{Os}(1)-\mathrm{C}(10)-\mathrm{O}(10)$ and Os(3)-C(10)-O(10) angles are 145.3(7) and 134.0(6) respectively. The $\operatorname{Os}(1), \operatorname{Os}(2)$, and $\mathrm{C}(10)$ plane is tilted $20.66^{\circ}$ out of the triosmium plane towards the alkyne ligand.

Each osmium atom is bonded to three terminal carbonyl groups. The individual Os-CO lengths range from $1.898(8)$ to $1.959(8) \AA$, while the $\mathrm{C}-\mathrm{O}$ distances range from $1.113(9)$ to $1.138(10) \AA$. The Os-C-O angles are in the range $175.5(8)-179.7(8)^{\circ}$ except the $\mathrm{Os}(1)-\mathrm{C}(2)-\mathrm{O}(2)$ bond which is $171.7(7)^{\circ}$ to show weak bonding with the adjacent Os atom $(\mathrm{Os}(2) \cdots \mathrm{C}(2)=2.78$ $\AA$ ) and could indicate a semibridging interaction. The analogous double cluster $\left[\mathrm{Os}_{3}(\mathrm{CO})_{10}\right]_{2}\left(\mu_{3}, \mu_{3}-\eta^{2}, \eta^{2}\right.$ $\left.\mathrm{C}_{14} \mathrm{H}_{20}\right)$ [6] and the diphenylacetylene complex $\mathrm{Os}_{3}(\mathrm{CO})_{10}\left(\mu_{3}-\eta^{2}-\mathrm{C}_{2} \mathrm{Ph}_{2}\right)$ [33] have been illustrated to exhibit two semibridging $\mathrm{CO}$ ligands with the two long Os $\cdots \mathrm{C}$ distances in the range $2.65-2.95 \AA$. In contrast, $\mathrm{Os}_{3}(\mathrm{CO})_{10}\left(\mu_{3}-\eta^{2}-\mathrm{C}_{2} \mathrm{Et}_{2}\right)$ [30] presents only one bridging $\mathrm{CO}$ ligand. We note that triosmium clusters containing both bridging and semibridging carbonyl ligands like $\mathbf{4}$ are rare in the literature $[34,35]$.

The coordination about the Mo atoms of $\mathbf{4}$ is essentially identical to that of 3-allyl except slight difference in interatomic bond distances and angles. The semibridging $\mathrm{Mo}(2)-\mathrm{C}(13)-\mathrm{O}(13)$ bond angle is $168.4(8)^{\circ}$, with $\mathrm{C}(13) \cdots \mathrm{Mo}(1)=2.80 \AA$.

\section{Conclusions}

Several heterometallic clusters of $\mathrm{Mo}_{2}, \mathrm{Co}_{2}$ and $\mathrm{M}_{3}$ $(\mathrm{M}=\mathrm{Fe}, \mathrm{Ru}, \mathrm{Os})$ species bridged by a 14-membered cyclodiyne ligand have been prepared. For each compound, although the spectroscopic data evidence little interactions between the two metal systems, it is promising to couple them inter- or intramolecularly by reduction, photoinduction, or thermoactivation methods to produce mixed-metal phane complexes.

The crystal structure of $\mathbf{4}$ is unique, and presents a bridging and a semibridging carbonyl bound to the $\mathrm{Os}_{3}$ cluster. On the basis of this and previous studies [6], it seems the $\left[\mathrm{Os}_{3}(\mathrm{CO})_{10}\left(\mu_{3}-\eta^{2}-\mathrm{C}_{14} \mathrm{H}_{20}\right)\right]$ moieties with either a bridging carbonyl, a bridging and a semi-bridging carbonyls, or two semi-bridging carbonyls are energetically comparable, and crystal packing forces [36] are probably the governing factors in determining their configurations.

\section{Acknowledgements}

We are grateful for support of this work by the National Science Council of Taiwan.

\section{References}

[1] (a) Y. Chi, D.K. Huang, Comprehensive Organometallic Chemistry II, vol. 10, Pergamon, Oxford, 1995, p. 85. (b) M.C. Comstock, J.R. Shapley, Coord. Chem. Rev. 143 (1995) 501. (c) D.F. Shriver, H.D. Kaesz, R.D. Adams, The Chemistry of Metal Cluster Complexes, VCH, New York, 1990.

[2] (a) W. Hübel, Organic Syntheses via Metal Carbonyls, WileyInterscience, New York, 1968, p. 273. (b) E. Sappa, A. Tiripicchio, P. Braunstein, Chem. Rev. 83 (1983) 203. (c) P.R. Raithby, M.J. Rosales, Adv. Inorg. Chem. Radiochem. 29 (1985) 169. (d) D.B. Crotjahn, Comprehensive Organometallic Chemistry II, vol. 12, Pergamon, Oxford, 1995, p. 741. (e) W.-Y. Yeh, C.-L. Ho, M.Y. Chiang, I.-T. Chen, Organometallics 16 (1997) 2698. (f) W.-Y. Yeh, S.-M. Peng, G.-H. Lee, J. Organomet. Chem. 572 (1999) 125.

[3] (a) M. Nakagawa, Topics in Nonbenzenoid Aromatic Chemistry, vol. 1, Hirokawa Publishing Co., Tokyo, 1973. (b) F. Sondheimer, Acc. Chem. Res. 5 (1972) 81. (c) R. Gleiter, Angew. Chem. Int. Ed. Engl. 31 (1992) 27. (d) R. Gleiter, M. Karcher, R. Jahn, H. Irngartinger, Chem. Ber. 121 (1988) 735. (e) R. Gleiter, W. Schäfer, Acc. Chem. Res. 23 (1990) 369.

[4] (a) F. Vögtle, Cyclophane Chemistry: Synthesis, Structures and Reactivity, Wiley, Chichester, UK, 1993. (b) D.J. Cram, J.M. Cram, Container Molecules and Their Guests, The Royal Society of Chemistry, London, 1994. (c) R. Gleiter, M. Merger, Angew. Chem. Int. Ed. Engl. 36 (1997) 2426. (d) E. KlosterJensen, J. Wirz, Angew. Chem. Int. Ed. Engl. 12 (1973) 671. (e) J.A. Gladysz, J.G. Fulcher, S.J. Lee, A.B. Bocarsley, Tetrahedron Lett. (1977) 3421. (f) R.B. King, A. Efraty, J. Am. Chem. Soc. 92 (1970) 6071. (g) R.B. King, A. Efraty, J. Am. Chem. Soc. 94 (1972) 3021. (h) R.B. King, M.N. Ackermann, J. Organomet. Chem. 67 (1974) 431. (i) G. Mehta, B. Viswanath, N. Sastry, E.D. Jemmis, D. Sivakumar, K. Reddy, A.C. Kunwar, Angew. Chem. Int. Ed. Engl. 31 (1992) 1488. (j) C.M. Adams, E.M. Holt, Organometallics 9 (1990) 980.

[5] (a) R. Gleiter, H. Stahr, B. Nuber, Organometallics 16 (1997) 646. (b) R. Gleiter, M. Merger, T. Oeser, H. Irngartinger, Tetrahedron Lett. 36 (1995) 4625. (c) R. Gleiter, H. Langer, V. Schehlmann, B. Nuber, Organometallics 14 (1995) 975. (d) R. Gleiter, H. Langer, B. Nuber, Angew. Chem. Int. Ed. Engl. 33 (1994) 1272. (e) R. Gleiter, G. Pflästerer, B. Nuber, J. Chem. Soc. Chem. Commun. (1993) 454. (f) R. Gleiter, G. Pflästerer, Organometallics 12 (1993) 1886. (g) R. Gleiter, V. Schehlmann, Angew. Chem. Int. Ed. Engl. 29 (1990) 1426.

[6] W.-Y. Yeh, M.-A. Hsu, S.-M. Peng, G.-H. Lee, Organometallics 18 (1999) 880.

[7] W.-Y. Yeh, M.-A. Hsu, S.-M. Peng, G.-H. Lee, Inorg. Chim. Acta, in press.

[8] D.F. Shriver, M.A. Drezdzon, The Manipulation of Air-Sensitive Compounds, second ed., Wiley, New York, 1986.

[9] B.F.G. Johnson, J. Lewis, Inorg. Synth. 13 (1972) 92.

[10] R Gleiter, R. Merger, B. Treptow, W. Wittwer, G. Pflästerer, Synthesis (1993) 558.

[11] D. Braga, F. Grepioni, E. Parisini, B.F.G. Johnson, C.M. Martin, J.G.M. Nairn, J. Lewis, M. Martinelli, J. Chem. Soc. Dalton Trans. (1993) 1891.

[12] (a) S. Aime, W. Dastrù, R. Gobetto, J. Krause, L. Violano, Inorg. Chim. Acta 235 (1995) 357. (b) G.A. Foulds, B.F.G. Johnson, J. Lewis, J. Organomet. Chem. 296 (1985) 147. 
[13] D.D. Perrin, W.L.F. Armarego, Purification of Laboratory Chemicals, third ed., Pergamon, Oxford, 1988.

[14] R.H. Blessing, Acta Crystallogr. Sect. A 51 (1995) 33.

[15] A.J. Deeming, Comprehensive Organometallic Chemistry II, vol. 7, Pergamon, Oxford, 1995, p. 685.

[16] S.P. Tunik, E.V. Grachova, V.R. Denisov, G.L. Starova, A.B. Nikol'skii, F.M. Dolgushin, A.I. Yanovsky, Y.T. Struchkov, J. Organomet. Chem. 536 (1997) 339.

[17] A.J. Deeming, S. Hasso, M. Underhill, J. Chem. Soc. Dalton Trans. (1975) 1614.

[18] A.J. Deeming, M.S.B. Felix, P.A. Bates, M.B. Hursthouse, J. Chem. Soc. Chem. Commun. (1987) 461.

[19] J. Bracker-Novak, S. Hajela, M. Lord, M. Zhang, E. Rosenberg, R. Gobetto, L. Milone, D. Osella, Organometallics 9 (1990) 1379 .

[20] S. Aime, G. Jannon, D. Osella, A.J. Arce, A.J. Deeming, J. Chem. Soc. Chem. Commun. (1984) 1987.

[21] M.R. Churchill, J.C. Fettinger, J.B. Keister, R.F. See, J.W. Ziller, Organometallics 4 (1985) 2112.

[22] A.J. Carty, N. Taylor, E. Sappa, Organometallics 7 (1988) 405.

[23] J.F. Blount, L.F. Dahl, C. Hoogzand, W. Hübel, J. Am. Chem. Soc. 88 (1966) 292.

[24] (a) G. Váradi, I. Vecsei, A. Vizi-Orosz, G. Pályi, A.G. Massey, J. Organomet. Chem. 114 (1976) 213. (b) G. Bor, S.F.A. Kettle, P.L. Stanghellini, Inorg. Chim. Acta 18 (1976) L18.
[25] R.S. Dickson, P.J. Fraser, Adv. Organomet. Chem. 12 (1974) 323.

[26] W.I. Bailey Jr., M.H. Chisholm, F.A. Cotton, L.A. Rankel, J. Am. Chem. Soc. 100 (1978) 5764.

[27] R.D. Adams, D.M. Collins, F.A. Cotton, Inorg. Chem. 13 (1974) 1086.

[28] R.J. Klingler, W.M. Butler, M.D. Curtis, J. Am. Chem. Soc. 100 (1978) 5034.

[29] J.P. Collman, L.S. Hegedus, J.R. Norton, R.G. Finke, Principles and Applications of Organotransition Metal Chemistry, University Science Books, Mill Valley, CA, 1987.

[30] E. Rosenberg, J. Bracker-Novak, R.W. Gellert, S. Aime, R. Gobetto, D. Osella, J. Organomet. Chem. 365 (1989) 163.

[31] W.-Y. Yeh, S.-L. Chen, S.-M. Peng, G.-H. Lee, J. Organomet. Chem. 461 (1993) 207.

[32] A.D. Clauss, J.R. Shapley, S.R. Wilson, J. Am. Chem. Soc. 103 (1981) 7387.

[33] (a) M. Tachikawa, J.R. Shapley, C.G. Pierpont, J. Am. Chem. Soc. 97 (1975) 7172. (b) C.G. Pierpont, Inorg. Chem. 16 (1977) 636.

[34] A.J. Deeming, A.M. Senior, J. Organomet. Chem. 439 (1992) 177.

[35] A.J. Deeming, Adv. Organomet. Chem. 26 (1986) 1.

[36] J.P. Glusker, K.N. Trueblood, Crystal Structure Analysis, Oxford University Press, London, 1985. 